\title{
COHOMOLOGY THEORIES ON LOCALLY CONFORMAL SYMPLECTIC MANIFOLDS*
}

\author{
HÔNG VÂN LÊ ${ }^{\dagger}$ AND JIŘI VANŽURA
}

\begin{abstract}
In this note we introduce primitive cohomology groups of locally conformal symplectic manifolds $\left(M^{2 n}, \omega, \theta\right)$. We study the relation between the primitive cohomology groups and the Lichnerowicz-Novikov cohomology groups of $\left(M^{2 n}, \omega, \theta\right)$, using and extending the technique of spectral sequences developed by Di Pietro and Vinogradov for symplectic manifolds. We discuss related results by many peoples, e.g. Bouche, Lychagin, Rumin, Tseng-Yau, in light of our spectral sequences. We calculate the primitive cohomology groups of a $(2 n+2)$-dimensional locally conformal symplectic nilmanifold as well as those of a l.c.s. solvmanifold. We show that the l.c.s. solvmanifold is a mapping torus of a contactomorphism, which is not isotopic to the identity.
\end{abstract}

Key words. Locally conformal symplectic manifold, Lichnerowicz-Novikov cohomology, primitive cohomology, spectral sequence.

AMS subject classifications. 53D35, 57R17, 55T99.

1. Introduction. A differentiable manifold $\left(M^{2 n}, \omega, \theta\right)$ provided with a nondegenerate 2 -form $\omega$ and a closed 1-form $\theta$ is called a locally conformal symplectic (l.c.s.) manifold, if $d \omega=-\omega \wedge \theta, d \theta=0$. The 1 -form $\theta$ is called the Lee form of $\omega$.

The class of l.c.s. manifolds has attracted strong interests among geometers in recent years. For instance, Vaisman showed that l.c.s. manifolds may be viewed as phase spaces for a natural generalization of Hamiltonian dynamics [30]. Bande and Kotschick showed that a pair composed of a contact manifold and a contactomorphism is naturally associated with a l.c.s. manifold [3] (see also Proposition 7.3 and Proposition 7.4 below). Furthermore, l.c.s. manifolds together with contact manifolds are the only transitive Jacobi manifolds [22, Remark 2.10]. It is also worth mentioning that locally conformal Kähler manifolds, a natural subclass of l.c.s. manifolds, are actively studied in complex geometry, e.g. see [15], [31].

Note that a l.c.s. manifold is locally conformal equivalent to a symplectic manifold, i.e. locally $\theta=d f$ and $\omega=e^{-f} \omega_{0}, d \omega_{0}=0$. By the Darboux theorem all symplectic manifolds of the same dimension are locally equivalent. Hence symplectic manifolds have only global invariants, and cohomological invariants are most natural among them. First (co)homological symplectic invariants were proposed in works by Gromov and Floer then followed by works by McDuff, Hofer and Salamon, Fukaya and Ono, Ruan, Tian, Witten and many others, including the first author of this note. This approach was based on the use of the theory of elliptic differential operators with purpose to make regular certain Morse (co)homology theory or the intersection theory on the infinite dimensional loop space on a symplectic manifold $M^{2 n}$, or on the space of holomorphic curves on $M^{2 n}$. This elliptic (co)homology theory has huge success, but the computational part of the theory is quite complicated. Almost at the same time, a "linear" symplectic cohomology theory has been developed, beginning with the paper by Brylinski [5], followed by Bouche [4], and then by other peoples (see

\footnotetext{
*Received October 9, 2012; accepted for publication October 10, 2013.

${ }^{\dagger}$ Institute of Mathematics of ASCR, Zitna 25, 11567 Praha 1, Czech Republic (hvle@math.cas.cz). H.V.L. was partially supported by the MSMT project "Eduard Čech Research Center" and by RVO:67985840.

¥Institute of Mathematics of ASCR, Zizkova 22, 61662 Brno, Czech Republic (vanzura@ipm.cz). J.V. was partially supported by the grant GAČR 201/08/0397 and by RVO:67985840.
} 
[11], [7], [29]). This theory is mostly motivated by analogues in Kähler geometry, the Dolbeault theory, and the cohomology theory for differential equations developed by Vinogradov and his school.

This linear symplectic cohomology theory has not yet drawn as much attention as it, to our opinion, should have. This is, probably, due to the fact that its potentially important applications are still in a phase of elaboration. The computational part of the linear theory seems to be not so complicated as in the elliptic theory, and this is an advantage of its.

In our paper we further develop the linear symplectic cohomology theory and extend it to l.c.s. manifolds. This is possible due to the validity of the Lefschetz decomposition for these manifolds. The main tool is the spectral sequence developed by Di Pietro and Vinogradov for symplectic manifolds, which has been now adapted and developed further for l.c.s. manifolds. We obtain new results and applications even for symplectic manifolds. In particular we unify various isolated results of the linear symplectic cohomology theory.

The structure of this note is as follows. In section 2 we introduce important linear operators on l.c.s. manifolds and study their properties. The Lefschetz filtration on the space $\Omega^{*}\left(M^{2 n}\right)$ of a l.c.s. manifold $\left(M^{2 n}, \omega, \theta\right)$ is discussed in section 3 together with differential operators respecting this filtration. Then we use this filtration to construct primitive cohomology groups for $\left(M^{2 n}, \omega, \theta\right)$ (Definition 3.9). Some simple properties of these groups are fixed in Proposition 3.10, Proposition 3.11 and Proposition 3.14, and their relations with previously proposed constructions are discussed (Remark 3.16). The spectral sequence associated with the Lefschetz filtration is studied in section 4 . In particular, its $E_{1}$-term is compared with the primitive (co)homological groups (Lemma 4.1) and the conformal invariance of this term is proven (Theorem 4.6). In section 5 we find some cohomological conditions on $\left(M^{2 n}, \omega, \theta\right)$ under which this spectral sequence stabilizes at the $E_{t}$-term (Theorems $5.2,5.8,5.13)$. The last of these theorems gives an answer to the Tseng-Yau question on relations between the primitive cohomology and the de Rham cohomology of a compact symplectic manifold. In section 6 we specialize the previous theory to Kähler manifolds and prove that for Kähler manifolds the spectral sequence stabilizes already at its first term (Theorem 6.2). In section 7 we compute the primitive cohomology groups of a compact $(2 n+2)$-dimensional l.c.s. nilmanifold and a compact 4-dimensional l.c.s. solvmanifold (Propositions 7.1, 7.2). We study some properties of primitive cohomology groups of l.c.s. manifolds associated with a co-orientation preserving contactomorphism (Proposition 7.4). In particular, we show that the compact l.c.s. solvmanifold is associated with a non-trivial co-orientation preserving contactomorphism (Theorem 7.6).

The cohomological theory developed in this note and its analogues have a much wider area of applications. For instance, it may be naturally adopted to the class of Poisson symplectic stratified spaces introduced in [17], since these singular symplectic spaces also enjoy the Lefschetz decomposition.

This project was started as a joint work of us with Alexandre Vinogradov based on H.V.L. preliminary results on l.c.s. manifolds. Alexandre Vinogradov has suggested us to extend the results to a slightly larger category of twisted symplectic manifolds. He made considerable contributions to improve the original text written by H.V.L., which we appreciate very much. Eventually we have noticed that our viewpoints are so different, so we decide to write the subject separately: in this paper we deal only with l.c.s. manifolds and Alexandre Vinogradov will deal with the extension to 
twisted symplectic manifolds.

2. Basic operators on a l.c.s. manifold. In this section we introduce and study basic linear differential operators acting on differential forms on a l.c.s. manifold $\left(M^{2 n}, \omega, \theta\right)$.

The first operator we need is the Lichnerowicz deformed differential $d_{\theta}$ : $\Omega^{*}\left(M^{2 n}\right) \rightarrow \Omega^{*}\left(M^{2 n}\right)$,

$$
d_{\theta}(\alpha):=d \alpha+\theta \wedge \alpha
$$

Clearly $d_{\theta}^{2}=0$ and $d_{\theta}(\omega)=0$. The resulting Lichnerowicz cohomology groups (also called the Novikov cohomology groups) are important conformal invariants of l.c.s. manifolds.

Recall that two l.c.s. forms $\omega$ and $\omega^{\prime}$ on $M^{2 n}$ are conformally equivalent, if $\omega^{\prime}= \pm\left(e^{f}\right) \omega$ for some $f \in C^{\infty}\left(M^{2 n}\right)$. In this case the corresponding Lee forms $\theta$ and $\theta^{\prime}$ are cohomologous: $\theta^{\prime}=\theta \mp d f$, hence $d_{\theta}$ and $d_{\theta^{\prime}}$ are gauge equivalent:

$$
d_{\theta^{\prime}}(\alpha)=\left(d_{\theta} \mp d f \wedge\right) \alpha=e^{ \pm f} d\left(e^{\mp f} \alpha\right) .
$$

It follows that $H^{*}\left(\Omega^{*}\left(M^{2 n}\right), d_{\theta}\right)$ and $H^{*}\left(\Omega^{*}\left(M^{2 n}\right), d_{\theta^{\prime}}\right)$ are isomorphic. The isomorphism $I_{f}: H^{*}\left(\Omega^{*}\left(M^{2 n}\right), d_{\theta}\right) \rightarrow H^{*}\left(\Omega^{*}\left(M^{2 n}\right), d_{\theta^{\prime}}\right)$ is given by the conformal transformation $[\alpha] \mapsto\left[ \pm e^{f} \alpha\right]$.

Note that $d_{\theta}$ does not satisfy the Leibniz property, unless $\theta=0$, since

$$
d_{\theta}(\alpha \wedge \beta)=d_{\theta} \alpha \wedge \beta+(-1)^{\operatorname{deg} \alpha} \alpha \wedge d \beta=d \alpha \wedge \beta+(-1)^{\operatorname{deg} \alpha} \alpha \wedge d_{\theta} \beta
$$

Thus the cohomology group $H^{*}\left(\Omega^{*}\left(M^{2 n}\right), d_{\theta}\right)$ does not have a ring structure, unless $\theta=0$. The formula $(2.2)$ also implies that $H^{*}\left(\Omega^{*}\left(M^{2 n}\right), d_{\theta}\right)$ is a $H^{*}(M, \mathbb{R})$ module.

Now let us consider the next basic linear operator

$$
L: \Omega^{*}\left(M^{2 n}\right) \rightarrow \Omega^{*}\left(M^{2 n}\right), \alpha \mapsto \omega \wedge \alpha .
$$

Substituting $\alpha:=\omega$ in (2.2) we obtain a nice relation between $d, L$ and $d_{\theta}$

$$
d_{\theta} L=L d
$$

The identity (2.4) suggests us to consider a family of operators $d_{k \theta}$, which we abbreviate as $d_{k}$ if no misunderstanding occurs. We derive immediately from (2.4)

$$
d_{k} L^{p}=L^{p} d_{k-p}
$$

The following Lemma is a generalization of (2.2) and it plays an important role in our study of the spectral sequences introduced in later sections. It is obtained by straightforward calculations, so we omit its proof.

Lemma 2.1. For any $\alpha, \beta \in \Omega^{*}\left(M^{2 n}\right)$ we have

$$
d_{k+l}(\alpha \wedge \beta)=d_{k} \alpha \wedge \beta+(-1)^{\operatorname{deg} \alpha} \alpha \wedge d_{l} \beta .
$$

Consequently

$$
d_{k} \alpha \wedge d_{l} \beta=d_{k+l}\left(\alpha \wedge d_{l} \beta\right)
$$


Formula (2.6) yields the induced map $H^{*}\left(\Omega^{*}\left(M^{2 n}\right), d_{k}\right) \times H^{*}\left(\Omega^{*}\left(M^{2 n}\right), d_{l}\right) \rightarrow$ $H^{*}\left(\Omega^{*}\left(M^{2 n}\right), d_{k+l}\right)$.

Denote by $G_{\omega}$ the section of the bundle $\Lambda^{2} T M^{2 n}$ such that for all $x \in M^{2 n}$ the linear map $i_{G_{\omega}(x)}: T_{x}^{*} M^{2 n} \rightarrow T_{x} M^{2 n}, V \mapsto i_{V}\left(G_{\omega}(x)\right)$, is the inverse of the map $I_{\omega}: T_{x} M^{2 n} \rightarrow T_{x}^{*} M^{2 n}, V \mapsto i_{V} \omega$. Clearly $G_{\omega}$ defines a bilinear pairing: $T^{*} M^{2 n} \times T^{*} M^{2 n} \rightarrow C^{\infty}\left(M^{2 n}\right)$. Denote by $\Lambda^{p} G_{\omega}$ the associated pairing: $\Lambda^{p}\left(T^{*} M\right) \times \Lambda^{p}\left(T^{*} M\right) \rightarrow C^{\infty}\left(M^{2 n}\right)$. The l.c.s. form $\omega$ and the associated bi-vector field $G_{\omega}$ define a l.c.s. star operator $*_{\omega}: \Omega^{p}\left(M^{2 n}\right) \rightarrow \Omega^{2 n-p}\left(M^{2 n}\right)$ as follows [5, §2.1].

$$
*_{\omega}: \Omega^{p}\left(M^{2 n}\right) \rightarrow \Omega^{2 n-p}\left(M^{2 n}\right), \beta \wedge *_{\omega} \alpha:=\Lambda^{p} G_{\omega}(\beta, \alpha) \wedge \frac{\omega^{n}}{n !},
$$

for all $\alpha, \beta \in \Omega^{p}\left(M^{2 n}\right)$. Using [5, Lemma 2.1.2] we get easily

$$
*_{\omega}^{2}=I d
$$

We define the l.c.s. adjoint $L^{*}$ of $L$ and the l.c.s. adjoint $\left(d_{k}\right)_{\omega}^{*}$ of $d_{k}$ with respect to the l.c.s. form $\omega$ as follows:

$$
\begin{gathered}
L^{*}: \Omega^{p}\left(M^{2 n}\right) \rightarrow \Omega^{p-2}\left(M^{2 n}\right), \alpha^{p} \mapsto-*_{\omega} L *_{\omega} \alpha^{p}, \\
\left(d_{k}\right)_{\omega}^{*}: \Omega^{p}\left(M^{2 n}\right) \rightarrow \Omega^{p-1}\left(M^{2 n}\right), \alpha^{p} \mapsto(-1)^{p} *_{\omega} d_{n+k-p} *_{\omega}\left(\alpha^{p}\right) .
\end{gathered}
$$

For symplectic manifolds our definition of $\left(d_{k}\right)_{\omega}^{*}$ agrees with the one in [35, §1], it is different from the one in [5, Theorem 2.2.1] by sign $(-1)$.

A section $g$ of the bundle $S^{2} T^{*} M^{2 n}$ is called a compatible metric, if there is an almost complex structure $J$ on $M^{2 n}$ such that $g(X, Y)=\omega(X, J Y)$. In this case $J$ is called a compatible almost complex structure. Recall that the Hodge operator $*_{g}$ is defined as follows

$$
*_{g}: \Omega^{p}\left(M^{2 n}\right) \rightarrow \Omega^{2 n-p}\left(M^{2 n}\right), \beta \wedge *_{g} \alpha:=\Lambda^{p} G_{g}(\beta, \alpha) \wedge \frac{\omega^{n}}{n !},
$$

where $G_{g} \in \Gamma\left(S^{2} T M^{2 n}\right)$ is the "inverse of $g$ ", i.e. it is defined in the same way as we define $G_{\omega}$ above: for all $x \in M^{2 n}$ the linear map $i_{G_{g}(x)}: T_{x}^{*} M^{2 n} \rightarrow T_{x} M, V \mapsto$ $i_{V}\left(G_{g}(x)\right)$, is the inverse of the map $I_{g}: T_{x} M \rightarrow T_{x}^{*} M, V \mapsto i_{V}(g)$. We also denote by $\Lambda^{p} G_{g}$ the associated pairing: $\Lambda^{p}\left(T^{*} M\right) \times \Lambda^{p}\left(T^{*} M\right) \rightarrow C^{\infty}\left(M^{2 n}\right)$ induced by $G_{g}$, (see also $\left[5\right.$, p.105] for comparing $\Lambda^{p} G_{\omega}$ with $\Lambda^{p} G_{g}$ ).

Using [32, Lemma 5.5] we get easily

$$
*_{g}^{2}\left(\alpha^{p}\right)=(-1)^{p} \alpha^{p} \text { for } \alpha^{p} \in \Omega^{p}\left(M^{2 n}\right) .
$$

Lemma 2.2. 1. The space of metrics compatible with a given l.c.s. form $\omega \in$ $\Omega^{2}\left(M^{2 n}\right)$ is contractible.

2. (cf. [32, chapter II, 6.2.1]) In the presence of a compatible metric $g$ on $M^{2 n}$ we have

$$
L^{*}=\Lambda
$$

where $\Lambda=\left(*_{g}\right)^{-1} L *_{g}$ is the adjoint of $L$ with respect to the metric $g$.

Proof. 1. The proof for the first assertion goes in the same way as for the case of symplectic manifolds, so we omit its proof. 
2. The second assertion of Lemma 2.2 is a simple consequence of the following

Lemma 2.3. [5, Theorem 2.4] Assume that $\left(M^{2 n}, J, g\right)$ is an almost Hermitian manifold and $\omega$ is the associated almost symplectic form. For $\alpha \in \Omega^{p, q}\left(M^{2 n}\right)$ we have

$$
*_{\omega}(\alpha)=\sqrt{-1}^{p-q} *_{g}(\alpha) .
$$

Here we extend $*_{\omega}$ and $*_{g} \mathbb{C}$-linearly on $\Omega^{*}\left(M^{2 n}\right) \otimes \mathbb{C}$.

This completes the proof of Lemma 2.2.

Let $\pi_{k}: \Omega^{*}\left(M^{2 n}\right) \rightarrow \Omega^{k}\left(M^{2 n}\right)$ be the projection. Denote $\sum_{i=0}^{2 n}(n-k) \pi_{k}$ by $A$. Using well-known identities in Kähler geometry for $(\Lambda, L, A)$, see e.g. [33, (IV), chapter I], [13, p.121], [32, Lemma 6.19], Lemma 2.2 implies immediately the following

Corollary 2.4. (cf. [21, §1], [35, Corollary 1.6]) On any l.c.s. manifold $\left(M^{2 n}, \omega, \theta\right)$ we have

$$
\begin{gathered}
L^{*}=i\left(G_{\omega}\right), \\
{\left[L^{*}, L\right]=A,[A, L]=-2 L,\left[A, L^{*}\right]=2 L^{*} .}
\end{gathered}
$$

The relation in $(2.16)$ shows that $\left(L^{*}, L, A\right)$ forms a $\mathfrak{s} l_{2}$-triple, which has many important consequences for l.c.s. manifolds.

Proposition 2.5. The following commutation relation hold

$$
L^{*}\left(d_{k}\right)_{\omega}^{*}=\left(d_{k-1}\right)_{\omega}^{*} L^{*} .
$$

Proof. Clearly (2.17) is obtained from (2.5) by applying the LHS and RHS of (2.5) the l.c.s. star operator on the left and on the right, taking into account (2.9).

3. Primitive forms and primitive cohomologies. In this section we introduce the notions of primitive forms and coeffective forms on a l.c.s. manifold $\left(M^{2 n}, \omega, \theta\right)$, using the linear operators $L$ and $L^{*}$ defined in the previous section. As in the symplectic case we obtain a Lefschetz decomposition of the space $\Omega^{*}\left(M^{2 n}\right)$ induced by primitive forms and coeffective forms together with various linear differential operators respecting this decomposition as well as an associated filtration (Propositions 3.5 and 3.7). The natural splitting of the introduced differential operators according to the Lefschetz decomposition leads to new cohomology groups of $\left(M^{2 n}, \omega, \theta\right)$ (Definition 3.9). In Propositions 3.10, 3.11, 3.14 we fix simple properties of these new cohomology groups. At the end of this section we compare our construction with related constructions in [21], [4], [25], [10], [35], [7], [8].

Definition 3.1. ([4], [35], cf. [33], [13]) An element $\alpha \in \Lambda^{k} T_{x}^{*} M^{2 n}, 0 \leq k \leq n$, is called primitive (or effective), if $L^{n-k+1} \alpha=0$. An element $\alpha \in \Lambda^{k} T_{x}^{*} M^{2 n}, n+1 \leq$ $k \leq 2 n$, is called primitive, if $\alpha=0$. An element $\beta \in \Lambda^{k} T_{x}^{*} M^{2 n}$ is called coeffective, if $L \beta=0$.

REMARK 3.2. 1. Wells in [34] refers to Lefschetz [18] and Weil [33] for the terminology "Lefschetz decomposition" and "primitive forms". Many mathematicians prefer "Lepage decomposition" and "effective forms" following Lepage in [19]. 
2. Clearly the notion of primitive form as well as the notion of coeffective form depends only on the conformal class of a l.c.s. form $\omega$.

The relation (2.16) between linear operators $L, L^{*}$ and $A$ leads to Lemma 3.3 below characterizing primitive forms and coeffective forms. The resulting Lefschetz decomposition of the space $\Lambda T^{*} M^{2 n}$ is a direct consequence of the $\mathfrak{s l}(2)$-module theory. Various variants of Lemma 3.3 for symplectic manifolds appeared in many works, beginning possibly with a paper by Lepage [19], with later applications in Kähler geometry [33], [13], [32], in a theory of second-order differential equations [21], in symplectic geometry [4], [35], etc..

We denote by $P_{x}^{k}\left(M^{2 n}\right)$ the set of primitive elements in $\Lambda^{k} T_{x}^{*} M^{2 n}$.

Lemma 3.3. 1. An element $\alpha \in \Lambda^{k} T_{x}^{*} M^{2 n}$ is primitive, if and only if $L^{*} \alpha=0$. 2. An element $\beta \in \Lambda^{k} T_{x}^{*} M^{2 n}$ is coeffective, if and only if $*_{\omega} \beta$ is primitive.

3. We have the following Lefschetz decomposition for $n \geq k \geq 0$ :

$$
\begin{gathered}
\Lambda^{n-k} T_{x}^{*} M^{2 n}=P_{x}^{n-k}\left(M^{2 n}\right) \oplus L P_{x}^{n-k-2}\left(M^{2 n}\right) \oplus L^{2} P_{x}^{n-k-4}\left(M^{2 n}\right) \oplus \cdots, \\
\Lambda^{n+k} T_{x}^{*} M^{2 n}=L^{k} P_{x}^{n-k}\left(M^{2 n}\right) \oplus L^{k+1} P_{x}^{n-k-2}\left(M^{2 n}\right) \oplus \cdots
\end{gathered}
$$

From Lemma 3.3 we get immediately

Corollary 3.4. 1. $L^{k}: \Lambda^{n-k} T_{x}^{*} M^{2 n} \rightarrow \Lambda^{n+k} T_{x}^{*} M^{2 n}$ is an isomorphism, for $0 \leq k \leq n$.

2. $L: \Lambda^{n-k-2} T_{x}^{*} M^{2 n} \rightarrow \Lambda^{n-k} T_{x}^{*} M^{2 n}$ is injective, for $k=-1,0,1, \cdots, n-2$.

It is useful to introduce the following notations. Denote by $P^{n-k} M^{2 n}$ the subbundle in $\Lambda T^{*} M^{2 n}$ whose fiber is $P_{x}^{n-k}\left(M^{2 n}\right)$. Let $\mathcal{P}^{n-k}\left(M^{2 n}\right) \subset \Omega^{n-k}\left(M^{2 n}\right)$ be the space of all smooth $(n-k)$-forms with values in $P^{n-k} M^{2 n}$. Elements of $\mathcal{P}^{n-k}\left(M^{2 n}\right)$ are called primitive $(n-k)$-forms. Let us set (cf. [29])

$$
\mathcal{L}^{s, r}:=L^{s} \mathcal{P}^{r} \text { for } 0 \leq s, r \leq n .
$$

Put $\mathcal{P}^{*}\left(M^{2 n}\right):=\oplus_{r} \mathcal{P}^{r}\left(M^{2 n}\right)$. Then Lemma 3.3 yields the following decompositions, which we call the first and second Lefschetz decompositions

$$
\Omega^{*}\left(M^{2 n}\right)=\mathcal{P}^{*}\left(M^{2 n}\right) \oplus L \Omega^{*}\left(M^{2 n}\right)=\bigoplus_{0 \leq 2 s+r \leq 2 n} \mathcal{L}^{s, r}
$$

Now we consider the interplay between the Lefschetz decompositions (3.4) and the linear differential operators introduced in the previous section. Iterating the action of $L$ on $K^{*}:=\Omega^{*}\left(M^{2 n}\right)$, we define the following filtration

$$
F^{0} K^{*}:=K^{*} \supset F^{1} K^{*}:=L K^{*} \supset \cdots \supset F^{k} K^{*}:=L^{k} K^{*} \supset \cdots \supset F^{n+1} K^{*}=\{0\} .
$$

Proposition 3.5. 1. The subset $F^{k} K^{*}$ is stable with respect to $d_{p}$ for all $k$ and $p$.

2. For any $\gamma \in \Omega^{1}\left(M^{2 n}\right)$ we have

$$
\gamma \wedge \mathcal{L}^{0, n-k} \subset \mathcal{L}^{0, n-k+1} \oplus \mathcal{L}^{1, n-k-1}
$$

Proof. 1. The first assertion of Proposition 3.5 follows from the identity $d_{p}\left(\omega^{k} \wedge\right.$ $\phi)=\omega^{k} \wedge d_{p-k} \phi$ for $\phi \in \Omega^{*}\left(M^{2 n}\right)$. 
2. Assume that $\alpha \in \mathcal{P}^{n-k}\left(M^{2 n}\right)=\mathcal{L}^{0, n-k}$. Then $L^{k+1}(\gamma \wedge \alpha)=\gamma \wedge L^{k+1}(\alpha)=0$. Taking into account the decomposition of $\gamma \wedge \alpha$ according to the second Lefschetz decomposition we obtain the second assertion of Proposition 3.5 immediately.

REMARK 3.6. 1. The relation $d_{p}\left(\omega^{k} \wedge \phi\right)=\omega^{k} \wedge d_{p-k} \phi$ can be also interpreted as an interplay between different filtered complexes $\left(F^{*} K^{*}, d_{k}\right)$ and $\left(F^{*} K^{*}, d_{p}\right)$. We shall investigate this interplay deeper in the next section.

2. We observe that the decompositions (3.1), (3.2) and (3.4) are compatible with the filtration (3.5) in the following sense. For any $p \geq 0$ and $0 \leq k \leq 2 n$ we have

$$
\begin{gathered}
F^{p} K^{*} \cap \Omega^{k}\left(M^{2 n}\right)=\oplus_{i=0}^{\left[\frac{k}{2}\right]-p} \mathcal{L}^{p+i, k-2 p-2 i} \text { if } k \geq 2 p, \\
F^{p} K^{*} \cap \Omega^{k}\left(M^{2 n}\right)=0 \text { if } k<2 p .
\end{gathered}
$$

The decomposition in (3.6) and (3.7) will be called the induced Lefschetz decomposition. It is important for understanding the spectral sequences introduced in the next section.

Proposition 3.7. The following inclusions hold

$$
\begin{aligned}
& d_{r} \mathcal{L}^{p, q-p} \subset \mathcal{L}^{p, q-p+1} \oplus \mathcal{L}^{p+1, q-p-1}, \\
& \left(d_{r}\right)_{\omega}^{*} \mathcal{P}^{n-k}\left(M^{2 n}\right) \subset \mathcal{P}^{n-k-1}\left(M^{2 n}\right) .
\end{aligned}
$$

Proof. Let $\beta \in \mathcal{P}^{q}\left(M^{2 n}\right)=\mathcal{L}^{0, q}$, so $L^{n-q+1} \beta=0$. We derive from (2.5)

$$
L^{n-q+1} d_{r} \beta=d_{r+n-q+1} L^{n-q+1} \beta=0 .
$$

Using (3.1) and (3.2) we get $d_{r} \beta \in \mathcal{P}^{q+1}\left(M^{2 n}\right)+L \mathcal{P}^{q-1}\left(M^{2 n}\right)$. This proves the inclusion (3.8) of Proposition 3.7 for $p=0$. The inclusion (3.8) for $p \neq 0$ follows from the particular case $p=0$ and the identity $d_{r} L^{p}=L^{p} d_{r-p}$.

Assume that $\beta \in \mathcal{P}^{n-k}\left(M^{2 n}\right)$. Taking into account (2.17) we obtain

$$
L^{*}\left(d_{r}\right)_{\omega}^{*} \beta=\left(d_{r-1}\right)_{\omega}^{*} L^{*} \beta,
$$

which is zero since $\beta$ is primitive. Hence $\left(d_{r}\right)_{\omega}^{*} \beta$ is also primitive. This proves (3.9) and completes the proof of Proposition 3.7.

Now we will show several consequences of Proposition 3.7. Denote by $\Pi_{p r}$ the projection $\Omega^{*}\left(M^{2 n}\right) \rightarrow \mathcal{P}^{*}\left(M^{2 n}\right)$ according to the Lefschetz decomposition in (3.4). Set

$$
d_{k}^{+}:=\Pi_{p r} d_{k}
$$

Using the first Lefschetz decomposition and Proposition 3.7 we decompose the operator $d_{k}: \Omega^{q}\left(M^{2 n}\right) \rightarrow \Omega^{q+1}\left(M^{2 n}\right)$ for $0 \leq q \leq n$ as follows (cf. [29]).

$$
d_{k}=d_{k}^{+}+L d_{k}^{-}
$$

where $d_{k}^{-}: \Omega^{q}\left(M^{2 n}\right) \rightarrow \Omega^{q-1}\left(M^{2 n}\right), 0 \leq q \leq n$. Note that $d_{k}^{-}$is well-defined, since $L: \Omega^{q-1}\left(M^{2 n}\right) \rightarrow \Omega^{q+1}\left(M^{2 n}\right)$ is injective. It is straightforward to check

$$
d_{k}^{+}\left(\mathcal{L}^{s, r}\right)=0 \text { if } s \geq 1 \text {, and } d_{k}^{-}\left(\mathcal{L}^{s, r}\right) \subset \mathcal{L}^{s, r-1} .
$$


Lemma 3.8. (cf. [29, Lemma 2.5, II]) The operators $d_{k}^{+}, d_{k-1}^{-}$satisfy the following properties

$$
\begin{array}{r}
\left(d_{k}^{+}\right)^{2}\left(\alpha^{q}\right)=0, \\
d_{k-1}^{-} d_{k}^{-}\left(\alpha^{q}\right)=0, \text { if } q \leq n, \\
\left(d_{k}^{-} d_{k}^{+}+d_{k-1}^{+} d_{k}^{-}\right) \alpha^{q}=0, \text { if } q \leq n-1, \\
\left(d_{k-1}\right)_{\omega}^{*}\left(d_{k}\right)_{\omega}^{*}\left(\alpha^{q}\right)=0 .
\end{array}
$$

Proof. We use the equality $d_{k}^{2}=0$ in the form $d_{k}\left(d_{k}^{+}+L d_{k}^{-}\right)=0$. Using (2.5) we get

$$
\left(d_{k}^{+}\right)^{2}+L\left(d_{k}^{-} d_{k}^{+}+d_{k-1}^{+} d_{k}^{-}\right)+L^{2} d_{k-1}^{-} d_{k}^{-}=0
$$

Now taking into account (3.12) and the injectivity of the operators $L: \Omega^{q}\left(M^{2 n}\right) \rightarrow$ $\Omega^{q+2}\left(M^{2 n}\right)$ and $L^{2}: \Omega^{q-1}\left(M^{2 n}\right) \rightarrow \Omega^{q+3}\left(M^{2 n}\right)$ for $q \leq n-1$ we obtain (3.13), (3.14), and (3.15).

Finally, (3.16) is a consequence of $d_{k}^{2}=0$ and $*_{\omega}^{2}=I d$.

Proposition 3.7 and Lemma 3.8 lead to new cohomology groups associated with a l.c.s. manifold $\left(M^{2 n}, \omega, \theta\right)$. We observe that $\mathcal{P}^{*}\left(M^{2 n}\right)$ is stable under the action of the operators $d_{k}^{+},\left(d_{k}\right)_{\omega}^{*}, d_{k}^{-}$.

Definition 3.9. Assume that $0 \leq q \leq n-1$.

The $k$-plus-primitive $q$-th cohomology group of $\left(M^{2 n}, \omega, \theta\right)$ is defined by

$$
H^{q}\left(\mathcal{P}^{*}\left(M^{2 n}\right), d_{k}^{+}\right):=\frac{\operatorname{ker} d_{k}^{+}: \mathcal{P}^{q}\left(M^{2 n}\right) \rightarrow \mathcal{P}^{q+1}\left(M^{2 n}\right)}{d_{k}^{+}\left(\mathcal{P}^{q-1}\left(M^{2 n}\right)\right)}
$$

The $k$-primitive $q$-th-cohomology group of $\left(M^{2 n}, \omega, \theta\right)$ is defined by

$$
H^{q}\left(\mathcal{P}^{*}\left(M^{2 n}\right),\left(d_{k}\right)_{\omega}^{*}\right):=\frac{\operatorname{ker}\left(d_{k}\right)_{\omega}^{*}: \mathcal{P}^{q}\left(M^{2 n}\right) \rightarrow \mathcal{P}^{q-1}\left(M^{2 n}\right)}{\left(d_{k+1}\right)_{\omega}^{*}\left(\mathcal{P}^{q+1}\left(M^{2 n}\right)\right)}
$$

The $k$-minus-primitive $q$-th cohomology group of $\left(M^{2 n}, \omega, \theta\right)$ is defined by

$$
H^{q}\left(\mathcal{P}^{*}\left(M^{2 n}\right), d_{k}^{-}\right):=\frac{\operatorname{ker} d_{k}^{-}: \mathcal{P}^{q}\left(M^{2 n}\right) \rightarrow \mathcal{P}^{q-1}\left(M^{2 n}\right)}{d_{k+1}^{-}\left(\mathcal{P}^{q+1}\left(M^{2 n}\right)\right)}
$$

Now we show few simple properties of the associated cohomology groups of a l.c.s. manifold. Note that the formula (3.21) below has been proved in [29, Proposition 3.15] for compact symplectic manifold $\left(M^{2 n}, \omega\right)$.

Proposition 3.10. Assume that $\left(M^{2 n}, \omega, \theta\right)$ is a l.c.s. manifold, $n \geq 2$.

1. Suppose that $[(k-1) \theta] \neq 0 \in H^{1}\left(M^{2 n}, \mathbb{R}\right)$. Then

$$
H^{1}\left(\mathcal{P}^{*}\left(M^{2 n}\right), d_{k}^{+}\right)=H^{1}\left(\Omega^{*}\left(M^{2 n}\right), d_{k}\right) .
$$

2. Suppose that $[(k-1) \theta]=0 \in H^{1}\left(M^{2 n}, \mathbb{R}\right)$. Then

$$
\begin{gathered}
H^{1}\left(\mathcal{P}^{*}\left(M^{2 n}\right), d_{k}^{+}\right)=H^{1}\left(\Omega^{*}\left(M^{2 n}\right), d_{\theta}\right) \text { if }[\omega] \neq 0 \in H^{2}\left(\Omega^{*}\left(M^{2 n}\right), d_{\theta}\right), \\
H^{1}\left(\mathcal{P}^{*}\left(M^{2 n}\right), d_{k}^{+}\right)=H^{1}\left(\Omega^{*}\left(M^{2 n}\right), d_{\theta}\right) \oplus R \text { if }[\omega]=0 \in H^{2}\left(\Omega^{*}\left(M^{2 n}\right), d_{\theta}\right),
\end{gathered}
$$


where $R$ is the 1-dimensional vector space generated by $\rho \in H^{1}\left(\mathcal{P}^{*}\left(M^{2 n}\right), d_{k}^{+}\right)$with $d_{k} \rho=\omega$.

Proof. 1. Assume that $0 \neq \alpha \in \mathcal{P}^{1}\left(M^{2 n}\right)$ and $d_{k}^{+} \alpha=0$, i.e. $[\alpha] \in$ $H^{1}\left(\mathcal{P}^{*}\left(M^{2 n}\right), d_{k}^{+}\right)$. Since $d_{k}^{+} \alpha=0$ we get $d_{k} \alpha=L f$, where $f \in C^{\infty}\left(M^{2 n}\right)$. Assume that $f \neq 0$. Using $d_{k}^{2} \alpha=0$ we derive $L d_{k-1} f=0$, which implies $d_{k-1} f=0$, since $L$ is injective. The equality $d_{k-1} f=0$ implies that $d_{k-1}$ is gauge equivalent to $d$. This contradicts the assumption of Proposition 3.10.1. Hence $f=0$. It follows $d_{k} \alpha=0$. Using $d_{k}^{+} h=d_{k} h$ for all $h \in \mathcal{P}^{0}\left(M^{2 n}\right)$ we obtain (3.20) immediately.

2. Now we assume that $[(k-1) \theta]=0 \in H^{1}\left(M^{2 n}, \mathbb{R}\right)$, or equivalently, $d_{k-1}$ is gauge equivalent to the canonical connection : $d_{k-1}=e^{h} d e^{-h}=d-d h \wedge$ for some $h \in C^{\infty}\left(M^{2 n}\right)$. In this case, as above, $d_{k} \alpha=L f$ implies $d_{k-1} f=0$, and hence $f=c e^{h}$.

a) Assume that (3.21) holds. If $c \neq 0$, then $\left[e^{h} \omega\right]=0 \in H^{2}\left(M^{2 n}, d_{k}\right)$. Since $(k-1) \theta=-d h$, the deformed differential $d_{\theta}-d h \wedge$ is gauge equivalent to $d_{k}$. It follows that $[\omega]=0 \in H^{2}\left(M^{2 n}, d_{\theta}\right)$. This contradicts the assumption of (3.21). Hence $c=0$. In this case we have $d_{k} \alpha=0$, and therefore $\left[e^{-h} \alpha\right] \in H^{1}\left(M^{2 n}, d_{\theta}\right)$. Taking into account $d_{k}^{+} f=d_{k} f$ for any $f \in \mathcal{P}^{0}\left(M^{2 n}\right)=\Omega^{0}\left(M^{2 n}\right)$, we obtain (3.21).

b) Assume that (3.22) holds. Then $e^{h} \omega=d_{k} \rho$ for some $\rho \in \Omega^{1}\left(M^{2 n}\right)$. In this case, $d_{k}(\alpha)=L f=\omega c e^{h}$ implies $d_{k}(\alpha-c \rho)=0$. We conclude that if $d_{k}^{+}(\alpha)=0$ then $\alpha=c \rho+\beta$ where $d_{k}(\beta)=0$. Clearly $[\beta] \in H^{1}\left(M^{2 n}, d_{k}\right)=H^{1}\left(M^{2 n}, d_{\theta}\right)$. This proves (3.22) and completes the proof of Proposition 3.10.

Proposition 3.11. (cf. [29, Lemma 2.7, part II]) Assume that $0 \leq k \leq n$. If $\alpha \in \mathcal{P}^{k}\left(M^{2 n}\right)$, then

$$
d_{r}^{-}\left(\alpha^{k}\right)=\frac{\left(d_{r}\right)_{\omega}^{*}\left(\alpha^{k}\right)}{n-k+1}
$$

Consequently $H^{k}\left(\mathcal{P}^{*}\left(M^{2 n}\right), d_{r}^{-}\right)=H^{k}\left(\mathcal{P}^{*}\left(M^{2 n}\right),\left(d_{r}\right)_{\omega}^{*}\right)$.

Proof. It suffices to prove (3.23) locally. Note that locally $d_{\theta}=d-d f \wedge$. In this case $(d-d f \wedge) \omega=0$ implies $\omega=e^{f} \omega_{0}$ with $d \omega_{0}=0$. Next, we compare $*_{\omega}$ and $*_{\omega_{0}}$, using (2.8) and the equality $G_{\omega}=e^{-f} G_{\omega_{0}}$.

$$
\beta^{k} \wedge *_{\omega} \alpha^{k}=\wedge^{k} G_{\omega}\left(\beta^{k}, \alpha^{k}\right) \wedge \frac{\omega^{n}}{n !}=\wedge^{k} e^{-k f} G_{\omega_{0}}\left(\beta^{k}, \alpha^{k}\right) e^{n f} \frac{\omega_{0}^{n}}{n !}=e^{(n-k) f} \beta^{k} \wedge *_{\omega_{0}} \alpha^{k},
$$

where $\beta^{k}, \alpha^{k} \in \Omega^{k}\left(M^{2 n}\right)$. It follows that

$$
*_{\omega}\left(\alpha^{k}\right)=e^{(n-k) f} *_{\omega_{0}}\left(\alpha^{k}\right) .
$$

Let $\alpha^{k} \in \mathcal{P}^{k}\left(M^{2 n}\right), 0 \leq k \leq n$. Denote by $(d)_{\omega_{0}}^{*}$ the symplectic adjoint of $d$ with respect to $\omega_{0}$. The formula (3.25) below, which is a partial case of (3.23) for symplectic manifold, has been proved in [29, Lemma 2.7, part II]. (We observe that their operator $d^{\Lambda}$ differs from our operator $(d)_{\omega_{0}}^{*}$ by sign $(-1)$.)

$$
d^{-}\left(\alpha^{k}\right)=\frac{(d)_{\omega_{0}}^{*} \alpha^{k}}{n-k+1}
$$

Using $d_{n+r-k} \alpha=e^{(n+r-k) f} d\left(e^{-(n+r-k) f} \alpha\right)$ we obtain from (3.24)

$$
\left(d_{r}\right)_{\omega}^{*}\left(\alpha^{k}\right)=(-1)^{k} *_{\omega} d_{n+r-k} *_{\omega}\left(\alpha^{k}\right)=
$$




$$
\begin{gathered}
=(-1)^{k} e^{(n-(2 n-k+1)) f} *_{\omega_{0}} e^{(n+r-k) f} d\left(e^{-(n+r-k) f}\left(e^{(n-k) f} *_{\omega_{0}} \alpha^{k}\right)=\right. \\
=(-1)^{k} e^{(r-1) f} *_{\omega_{0}} d\left(e^{-r f} *_{\omega_{0}} \alpha^{k}\right)= \\
=e^{-f}\left((d)_{\omega_{0}}^{*} \alpha^{k}+(-1)^{k} *_{\omega_{0}}(-r) d f \wedge *_{\omega_{0}} \alpha^{k}\right)
\end{gathered}
$$

Substituting $r=0$, we derive from (3.27)

$$
(d)_{\omega_{0}}^{*}\left(\alpha^{k}\right)=e^{f}(d)_{\omega}^{*} \alpha^{k}
$$

Next we compare $d_{r}^{-}$with $d^{-}$.

$$
d_{r}\left(\alpha^{k}\right)=e^{r f} d\left(e^{-r f} \alpha^{k}\right)=e^{r f}\left[d^{+}\left(e^{-r f} \alpha^{k}\right)+\omega_{0} \wedge d^{-} e^{-r f} \alpha^{k}\right] .
$$

Since the Lefschetz decomposition of $\Omega\left(M^{2 n}\right)$ is invariant under conformal transformations we obtain from (3.29)

$$
d_{r}^{-}\left(\alpha^{k}\right)=e^{(r-1) f} d^{-}\left(e^{-r f} \alpha^{k}\right) .
$$

Combining (3.30) with (3.25) we conclude that

$$
d_{r}^{-}\left(\alpha^{k}\right)=e^{(r-1) f} \frac{(d)_{\omega_{0}}^{*}\left(e^{-r f} \alpha^{k}\right)}{n-k+1}
$$

Taking into account (3.28) and (3.26), we derive from (3.31)

$$
d_{r}^{-}\left(\alpha^{k}\right)=e^{r f} \frac{(d)_{\omega}^{*} e^{-r f} \alpha^{k}}{n-k+1}=\frac{\left(d_{r}\right)_{\omega}^{*} \alpha^{k}}{n-k+1} .
$$

This proves (3.23).

Clearly the second assertion of Proposition 3.11 follows from (3.23). This completes the proof of Proposition 3.11.

Let $J$ be a compatible almost complex structure on a l.c.s. manifold $\left(M^{2 n}, \omega, \theta\right)$. The complexified space $T_{\mathbb{C}}^{*}\left(M^{2 n}\right):=\left(T^{*}\left(M^{2 n}\right) \otimes \mathbb{C}\right.$ is decomposed into eigen-subspaces $T^{p, q}\left(M^{2 n}\right)$. Let $\Pi^{p, q}: T_{\mathbb{C}}^{*}\left(M^{2 n}\right) \rightarrow T^{p, q}\left(M^{2 n}\right)$ be the projection. Set

$$
\mathcal{J}:=\sum_{p, q}(\sqrt{-1})^{p-q} \Pi^{p, q} .
$$

In what follows we want to apply the Hodge theory to compact l.c.s. manifolds $\left(M^{2 n}, \omega, \theta\right)$ provided with a compatible metric $g$. First we derive a formula for the formal adjoint $\left(d_{l}^{+}\right)^{*}$ of $d_{l}^{+}: \mathcal{P}^{*}\left(M^{2 n}\right):=\mathcal{P}^{*}\left(M^{2 n}\right) \rightarrow \mathcal{P}^{*}\left(M^{2 n}\right) \subset \Omega^{*}\left(M^{2 n}\right)$. For any operator $D$ acting on a subbundle $E \subset \Omega^{*}\left(M^{2 n}\right)$ we denote by $(D)^{*}$ the formal adjoint of $D$.

Lemma 3.12. For any $\alpha \in \mathcal{P}^{*}\left(M^{2 n}\right)$ we have

$$
\left(d_{l}^{+}\right)^{*}(\alpha)=-*_{g}\left(d_{-l}\right) *_{g}(\alpha) .
$$

Proof. First, we want to compute the formal adjoint $\left(d_{l}\right)^{*}$ of $d_{l}=d+l \theta \wedge$ : $\Omega^{*}\left(M^{2 n}\right) \rightarrow \Omega^{*}\left(M^{2 n}\right)$. It is known that $[32, \S 5.1 .2]$

$$
(d)^{*}=-*_{g} d *_{g} .
$$


Since $\theta \wedge$ is the symbol of $d$ we derive from (3.34)

$$
(l \theta \wedge)^{*}=*_{g} l \theta \wedge *_{g} .
$$

It follows from (3.34) and (3.35)

$$
\left(d_{l}\right)^{*}=-*_{g} d_{-l} *_{g} .
$$

Using (2.14) we get for $\alpha \in \mathcal{P}^{*}\left(M^{2 n}\right)$

$$
\left(L d_{l}^{-}\right)^{*}(\alpha)=\left(d_{l}^{-}\right)^{*} \Lambda(\alpha)=0 .
$$

It follows from (3.36) and (3.37) that for $\alpha \in \mathcal{P}^{*}\left(M^{2 n}\right)$

$$
\left(d_{l}^{+}\right)^{*}(\alpha)=-*_{g}\left(d_{-l}\right) *_{g}(\alpha) .
$$

This proves $(3.33)$, which is consistent with $\left[29,(3.2)\right.$, part II], if $\left(M^{2 n}, \omega\right)$ is a symplectic manifold.

Lemma 3.13. (cf. [29, Lemma 3.4, part II]) Let $J$ be a compatible almost complex structure on a l.c.s. manifold $\left(M^{2 n}, \omega, \theta\right), g$ the associated compatible metric and $* g$ the Hodge star operator with respect to $g$. Then for $\alpha^{k}, \alpha^{k-1} \in \mathcal{P}^{*}\left(M^{2 n}\right), 0 \leq k \leq n$, we have

$$
\begin{array}{r}
\mathcal{J}\left(d_{l}^{+}\right)^{*} \mathcal{J}^{-1}\left(\alpha^{k}\right)=(n-k+1) d_{-l+k-n}^{-}\left(\alpha^{k}\right), \\
\mathcal{J} d_{l}^{+} \mathcal{J}^{-1}\left(\alpha^{k-1}\right)=(n-k+1)\left(d_{-l+k-n}^{-}\right)^{*}\left(\alpha^{k-1}\right) .
\end{array}
$$

Proof. Using [5, Theorem 2.4], see also Lemma 2.3, we get easily

$$
\mathcal{J}=*_{g} *_{\omega}
$$

By (2.13) we derive from (3.41)

$$
\mathcal{J}^{-1}\left(\alpha^{k}\right)=*_{\omega} *_{g}(-1)^{k}\left(\alpha^{k}\right)
$$

Combining (3.42) with (3.41), (3.33) and applying (2.11), (2.13) again we obtain

$$
\begin{array}{r}
\mathcal{J}\left(d_{l}^{+}\right)_{g}^{*} \mathcal{J}^{-1}\left(\alpha^{k}\right)=(-1)^{k+1} *_{g} *_{\omega} *_{g} d_{-l} *_{g} *_{\omega} *_{g}\left(\alpha^{k}\right)= \\
=(-1)^{k+1} *_{g}^{2}\left(d_{-l+k-n}\right)_{\omega}^{*}\left(\alpha^{k}\right)=\left(d_{-l+k-n}\right)_{\omega}^{*}\left(\alpha^{k}\right),
\end{array}
$$

since $*_{\omega} *_{g}=*_{g} *_{\omega}$. Using (3.23) we derive (3.40) immediately from (3.43). Clearly (3.40) follows from (3.39), since they are adjoint. This completes the proof of Lemma 3.13.

The following Proposition is a generalization of [29, Proposition 3.5, part II].

Proposition 3.14. Let $\left(M^{2 n}, \omega, \theta\right)$ be a compact l.c.s manifold. Then there is $H^{k}\left(\mathcal{P}^{*}\left(M^{2 n}\right), d_{l}^{+}\right)=H^{k}\left(\mathcal{P}^{*}\left(M^{2 n}\right),\left(d_{-l+k-n}\right)_{\omega}^{*}\right)$ for all $l \in \mathbb{Z}$ and $0 \leq k \leq n-1$.

Proof. First we note that all the operators $d_{l}^{+}, d_{l}^{-}$and $\left(d_{l}\right)_{\omega}^{*}$ restricted to the space $\mathcal{P}^{*}\left(M^{2 n}\right)$ are elliptic operators. This observation is a consequence of the theorem by Bouche who proved that the complex of coeffective forms on a symplectic manifold $M^{2 n}$ is elliptic in dimension greater than $n$ [4]. Indeed, the complex $\left(\mathcal{P}^{*}\left(M^{2 n}\right),(d)_{\omega}^{*}\right)$ is dual to the complex of coeffective forms, see also Remark 3.16.1 below. Thus $\left(d_{l}\right)_{\omega}^{*}$ 
acting on $\mathcal{P}^{*}\left(M^{2 n}\right)$ is an elliptic operator, since $\left(d_{l}\right)_{\omega}^{*}$ has the same symbol as $(d)_{\omega}^{*}$. Taking $(3.23),(3.40)$ and (3.39) into account we prove the ellipticity of $d_{l}^{-}$and $d_{l}^{+}$ acting on $\mathcal{P}^{*}\left(M^{2 n}\right)$. In [29, Proposition 2.8 part II] the authors give another proof of the ellipticity of these operators.

Now Proposition 3.14 follows easily from Lemma 3.13 using the Hodge theory.

Corollary 3.15. Assume that $\left(M^{2 n}, \omega, \theta\right)$ is a connected compact l.c.s. manifold. Then $H^{0}\left(\mathcal{P}^{*}\left(M^{2 n}\right), d_{k}^{+}\right)=0$ if $d_{k}$ is not gauge equivalent to the canonical differential $d=d_{0}$, ore equivalently $[k \theta] \neq 0 \in H^{1}\left(M^{2 n}, \mathbb{R}\right)$. If otherwise, then $H^{0}\left(\mathcal{P}^{*}\left(M^{2 n}\right), d_{k}^{+}\right)=H^{0}\left(\mathcal{P}^{*}\left(M^{2 n}\right),\left(d_{k}\right)_{\omega}^{*}\right)=H^{0}\left(\mathcal{P}^{*}\left(M^{2 n}\right), d_{k}^{-}\right)=\mathbb{R}$.

Proof. Note that $\mathcal{P}^{0}\left(M^{2 n}\right)=\Omega^{0}\left(M^{2 n}\right)$ and $d_{k}^{+}=d_{k}$, which implies the first assertion of Proposition 3.15 immediately. The second assertion of Proposition 3.15 follows from Proposition 3.11 and Proposition 3.14, taking into account the equalities $H^{0}\left(\mathcal{P}^{*}\left(M^{2 n}\right), d\right)=H^{0}\left(M^{2 n}, \mathbb{R}\right)=\mathbb{R}$.

REMARK 3.16. 1. Let $\left(M^{2 n}, \omega, \theta\right)$ be a l.c.s. manifold. The symplectic star operator $*_{\omega}$ provides an isomorphism between the space $\mathcal{P}^{*} M^{2 n}$ of primitive forms and the space $\mathcal{C}^{*} M^{2 n}$ of coeffective forms. Thus $H^{*}\left(\mathcal{P}^{*}\left(M^{2 n}\right)\right)$ is isomorphic to $H^{*}\left(\mathcal{C}^{*} M^{2 n}, d_{\theta}\right)$. The latter cohomology groups for symplectic manifolds have been introduced by Bouche [4]. A variant of the effective cohomology groups for contact manifolds has been introduced (and computed) by Lychagin [21] already in 1979. Later, a modification of this complex for contact manifolds has been considered by Rumin independently [25]. Chinea, Marrero and Leo generalized the construction of effective cohomology groups for Jacobi manifolds [6].

2. Note that the groups $H^{q}\left(\mathcal{P}^{*}\left(M^{2 n}\right), d_{k}^{+}\right)$have the following simple interpretation. We consider the differential ideal $L\left(\Omega^{*}\left(M^{2 n}\right)\right) \subset \Omega^{*}\left(M^{2 n}\right)$. The quotient $\Omega^{*}\left(M^{2 n}\right) / L\left(\Omega^{*}\left(M^{2 n}\right)\right)$ is isomorphic to the space $\mathcal{P}^{*}\left(M^{2 n}\right)$, and the differential $d_{k}$ induces the differential $d_{k}^{+}$on the quotient complex.

3. The plus-primitive cohomology groups and the minus-primitive cohomology groups for symplectic manifolds have been introduced by Tseng and Yau [29, part II].

4. Below we shall show a deeper relation between these new cohomology groups and the twisted cohomology groups $H^{*}\left(M^{2 n}, d_{k}\right)$ using the spectral sequence introduced in the next section.

5. Let $\left(M^{2 n+1}, \alpha\right)$ be a contact manifold. Then its symplectization $M^{2 n+2}:=$ $M^{2 n+1} \times \mathbb{R}$ is supplied with a symplectic form $\omega(x, t)=\exp ^{t}(d \alpha+d t \wedge \alpha)=\tilde{\alpha}$. Denote by $i: M^{2 n+1} \rightarrow M^{2 n+2}$ the embedding $x \mapsto(x, 0)$. We observe that the restriction of the filtration on $\left(M^{2 n+2}, d \tilde{\alpha}\right)$ to $i\left(M^{2 n+1}\right)$ coincides with the filtration introduced by Lychagin [21].

6. Note that any symplectic manifold $\left(M^{2 n}, \omega\right)$ is a Poisson manifold equipped with the Poisson bivector $G_{\omega}$. Associated with a Poisson bivector $\Lambda$ on a Poisson manifold $M$ there are two differential complexes. The first one is the complex of multivector fields on $M$ equipped with the differential $\Lambda$ acting via the Schouten bracket. It has been introduced by Lichnerowicz and the associated cohomology group is called the Lichnerowicz-Poisson cohomology of $M$ [20], [10]. The second differential complex is the complex of differential forms on $M$ equipped with the differential $\delta=[i(\Lambda), d]$ where $i(\Lambda)$ is the contraction with $\Lambda$. This complex has been introduced by Kozsul in [16] and it is called the canonical Poisson homology of $M$ [5]. If $M^{2 n}$ is symplectic then $G_{\omega} \in \operatorname{End}\left(T^{*} M^{2 n}, T M^{2 n}\right)$ induces an isomorphism between the de Rham cohomology and Lichnerowicz-Poisson cohomology [10, Theorem 6.1], and the symplectic star operator provides an isomorphism between the de Rham cohomology 
and the canonical Poisson homology [5]. In [10] the authors consider the coeffective Lichnerowicz-Poisson cohomology groups on a Poisson manifold, which are isomorphic to the coeffective symplectic groups introduced by Bouche [4] if the Poisson structure is symplectic.

4. Spectral sequences on a l.c.s. manifold. In this section, for any integer $k$, we construct a spectral sequence associated with the filtered complex $\left(F^{*} K^{*}, d_{k}\right)$ on a l.c.s. manifold $\left(M^{2 m}, \omega, \theta\right)$. We compare the $E_{1}$-term of this spectral sequence with the primitive cohomology group $H^{*,+}\left(\mathcal{P}^{*}\left(M^{2 n}\right), d_{k}\right)$ introduced in the previous section (Lemma 4.1). We show the existence of a long exact sequence connecting the $E_{1}$-term of this spectral sequence with the Lichnerowicz-Novikov cohomology groups $H^{*}\left(\Omega^{*}\left(M^{2 n}\right), d_{k+p}\right)$ for appropriate $p$, which will be denoted by $H_{k+p}^{*}\left(M^{2 n}\right)$ (Theorem 4.3 , Proposition 4.5$)$. We prove that the $E_{1}$-term of our spectral sequence is a conformal invariant of $\left(M^{2 n}, \omega, \theta\right)$, moreover, the $E_{1}$-terms associated with $\left(M^{2 n}, \omega, \theta\right)$ and $\left(M^{2 n}, \omega^{\prime}, \theta\right)$ are isomorphic, if $\omega^{\prime}=\omega+d_{\theta} \tau$ (Theorem 4.6).

In Proposition 3.5 of the previous section we proved that $\left(F^{*} K^{*}, d_{k}\right)$ is a filtered complex. Let us study the spectral sequence $\left\{E_{k, r}^{p, q}, d_{k, r}: E_{k, r}^{p, q} \rightarrow E_{k, r}^{p+r, q-r+1}\right\}$ of this filtered complex, first introduced by Di Pietro and Vinogradov for symplectic manifolds in [7]. We refer the reader to [23], [13] for an introduction into the theory of spectral sequences associated with a filtration. The initial term $E_{k, 0}^{p, q}$ of this spectral sequence is defined as follows,

$$
E_{k, 0}^{p, q}=F^{p} K^{p+q} / F^{p+1} K^{p+q} .
$$

Using the induced Lefschetz decomposition (3.6), (3.7), taking into account the injectivity of the map $L^{p}: \Omega^{q-p}\left(M^{2 n}\right) \rightarrow \Omega^{q+p}\left(M^{2 n}\right)$, we get for all $k \in \mathbb{Z}$

$$
\begin{aligned}
E_{k, 0}^{p, q} \cong \mathcal{L}^{p, q-p} \cong \mathcal{L}^{0, q-p} \text { if } n \geq q \geq p \geq 0, \\
E_{k, 0}^{p, q}=0 \text { otherwise } .
\end{aligned}
$$

Since $E_{k, l}^{p, q}$ is a quotient of $E_{k, l-1}^{p, q}$, in view of (4.3), any term $E_{k, l}^{p, q}$ written below, if without explicit condition on $p$ and $q$, is always under the assumption $0 \leq p \leq q \leq n$.

Now let us go to the next term $E_{k, 1}^{p, q}$ of our spectral sequence. Recall that $d_{k, 0}$ : $E_{k, 0}^{p, q} \rightarrow E_{k, 0}^{p, q+1}$ is obtained from the differential $d_{k}$ by passing to the quotient:

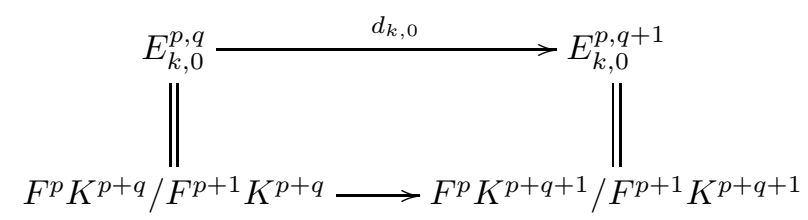

Let us write $d_{k, 0}$ explicitly using (4.2) and (4.3). Since $d_{k} L^{p}=L^{p} d_{k-p}$, using (4.1), (4.2), (4.3) and (3.11) we have for any $\alpha \in E_{k, 0}^{p, q}$ with $n \geq q \geq p \geq 0$

$$
d_{k, 0}(\alpha)=\left[L^{p}\left(d_{k-p}^{+}+L d_{k-p}^{-}\right)(\tilde{\alpha})\right]=\left[L^{p} d_{k-p}^{+}(\tilde{\alpha})\right] \in E_{k, 0}^{p, q+1},
$$

where $\tilde{\alpha} \in \mathcal{L}^{0, q-p}$ is a representative of $\alpha \in E_{k, 0}^{p, q}$ by $(4.2)$. Since $L^{p}: \mathcal{L}^{0, q-p} \rightarrow \mathcal{L}^{p, q-p}$ is an isomorphism, if $0 \leq p \leq q \leq n$ by (4.2), we rewrite (4.5) as follows

$$
\begin{array}{r}
d_{k, 0}: E_{k, l}^{p, q}=\mathcal{L}^{0, q-p} \rightarrow E_{k, 0}^{p, q+1}=\mathcal{L}_{k, 0}^{0, q+1-p}, \tilde{\alpha} \mapsto d_{k-p}^{+} \tilde{\alpha} \\
\text { if } 0 \leq p \leq q \leq n .
\end{array}
$$


LEMMA 4.1. The term $E_{k, 1}^{*, *}$ of the spectral sequence $\left\{E_{k, r}^{p, q}, d_{k, r}\right\}$ is determined by the following relations

$$
\begin{gathered}
E_{k, 1}^{p, q}=H^{q-p}\left(\mathcal{P}^{*}\left(M^{2 n}\right), d_{k-p}^{+}\right) \text {if } 0 \leq p \leq q \leq n-1, \\
E_{k, 1}^{p, n}=\frac{\mathcal{P}^{n-p}\left(M^{2 n}\right)}{d_{k-p}^{+}\left(\mathcal{P}^{n-p-1}\left(M^{2 n}\right)\right)}, \text { if } 0 \leq p \leq n, \\
E_{k, 1}^{p, q}=0 \text { otherwise. }
\end{gathered}
$$

Proof. Clearly (4.7) is a consequence of (4.6). Next using (4.5) and the identity $L^{p}\left(\mathcal{P}^{n-p+1}\left(M^{2 n}\right)\right)=0$, we obtain $d_{k, 0}\left(E_{k, 0}^{p, n}\right)=0$. Hence

$$
E_{k, 1}^{p, n}=\frac{L^{p}\left(\mathcal{P}^{n-p}\left(M^{2 n}\right)\right)}{L^{p}\left(d_{k-p}^{+}\left(\mathcal{P}^{n-p-1}\left(M^{2 n}\right)\right)\right)} .
$$

Since $L^{p}: \Omega^{n-p}\left(M^{2 n}\right) \rightarrow \Omega^{n+p}\left(M^{2 n}\right)$ is injective, (4.10) implies (4.8).

The last relation (4.9) in Lemma 4.1 follows from (4.3). This completes the proof of Lemma 4.1.

Next we define the following diagram of chain complexes

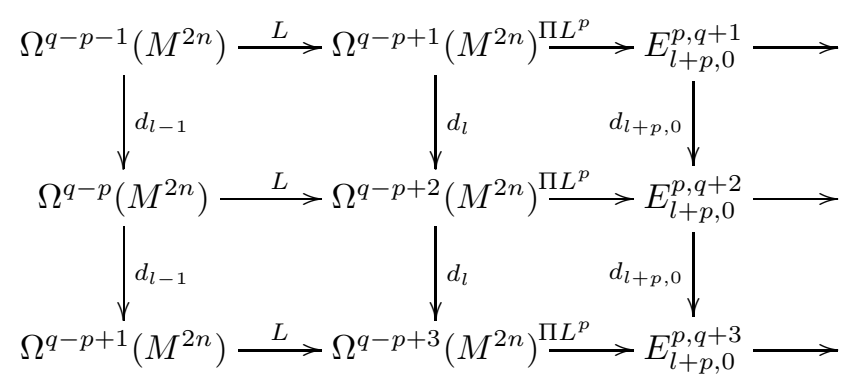

Here the map $\Pi L^{p}$ associates with each element $\beta \in \Omega^{q-p+1}\left(M^{2 n}\right)$ the element $\left[L^{p} \beta\right] \in E_{l+p, 0}^{p, q+1}$. Recall that $d_{l} \circ L=L \circ d_{l-1}$. Thus the upper part of the above diagram is commutative. The lower part of the diagram is also commutative, since

$$
d_{l+p} L^{p}=L^{p} d_{l}
$$

Summarizing we have the following sequence of chain complexes

$$
0 \rightarrow\left(\Omega^{q-(p+1)}\left(M^{2 n}\right), d_{l-1}\right) \stackrel{L}{\rightarrow}\left(\Omega^{q+1-p}\left(M^{2 n}\right), d_{l}\right) \stackrel{\Pi L^{p}}{\rightarrow}\left(E_{l+p, 0}^{p, q+1}, d_{l+p, 0}\right) \rightarrow 0 .
$$

Set $\Omega^{-1}\left(M^{2 n}\right):=0$.

Lemma 4.2. The sequence (4.11) of chain complexes is exact for $0 \leq p \leq q \leq$ $n-1$.

Proof. For $0 \leq p \leq q \leq n$ the operator $L: \Omega^{q-p-1}\left(M^{2 n}\right) \rightarrow \Omega^{q-p+1}\left(M^{2 n}\right)$ is injective by Lemma 3.4, so the sequence (4.11) is exact at $\Omega^{q-(p+1)}\left(M^{2 n}\right)$. The exactness at $\Omega^{q+1-p}\left(M^{2 n}\right)$ follows easily from Corollary 3.4, taking into account (4.2). The exactness at $E_{l+p, 0}^{p, q+1}$ follows directly from the definition (4.1) of $E_{l+p, 0}^{p, q}$. 
As a consequence of Lemma 4.2, using the general theory of homological algebra, see e.g. [12, Chapter $1, \S 6]$, we get immediately

THEOREM 4.3. The following long sequence is exact for $0 \leq p \leq q \leq n-1$

$$
\cdots \rightarrow H_{l}^{q-p}\left(M^{2 n}\right) \stackrel{\bar{L}^{p}}{\rightarrow} E_{l+p, 1}^{p, q} \stackrel{\delta_{p, q}}{\rightarrow} H_{l-1}^{q-(p+1)}\left(M^{2 n}\right) \stackrel{\bar{L}}{\rightarrow} H_{l}^{q+1-p}\left(M^{2 n}\right) \stackrel{\bar{L}^{p}}{\rightarrow} E_{l+p, 1}^{p, q+1} \rightarrow \cdots
$$

REMARK 4.4. 1. Theorem 4.3 is a generalization of [7, Theorem 1] stated for symplectic manifolds.

2. Let us write the connecting homomorphism $\delta_{p, q}$ explicitly. If $\alpha \in$ $H^{q-p}\left(\mathcal{P}^{*}\left(M^{2 n}\right), d_{l}^{+}\right)=E_{l+p, 1}^{p, q}$, see $(4.7)$, then $d_{l} \tilde{\alpha}=L \eta$ for any representative $\tilde{\alpha} \in \mathcal{P}^{q-p}\left(M^{2 n}\right)$ of $\alpha$. By the definition of connecting homomorphism, see also [7, §3], $\delta_{p, q}(\alpha)=[\eta] \in H_{l-1}^{q-(p+1)}\left(M^{2 n}\right)$. Since $d_{l}^{+} \tilde{\alpha}=0, d_{l} \tilde{\alpha}=L d_{l}^{-} \tilde{\alpha}$, so $\eta=d_{l}^{-} \tilde{\alpha}$. Thus we get

$$
\delta_{p, q} \alpha=\left[d_{l}^{-} \tilde{\alpha}\right] \in H_{l-1}^{q-(p+1)}\left(M^{2 n}\right) .
$$

3. Let us write the operator $\bar{L}^{p}$ explicitly. Assume that $[\beta] \in H_{l}^{q-p}\left(M^{2 n}\right)$, $\beta \in \Omega^{q-p}\left(M^{2 n}\right)$. Set

$$
\beta_{p r}:=\Pi_{p r} \beta
$$

- the primitive component of $\beta$ in the first Lefschetz decomposition. Since $d_{l} \beta=0$, we have $d_{l}^{+} \beta_{p r}=0$. Thus the image $\bar{L}^{p}[\beta]=\left[L^{p} \beta\right] \in E_{l+p, 1}^{p, q}=H^{q-p}\left(\mathcal{P}^{*}\left(M^{2 n}\right), d_{l}^{+}\right)$ has a representative $\beta_{p r} \in \mathcal{P}^{q-p}\left(M^{2 n}\right)$ with $d_{l}^{+} \beta_{p r}=0$. Summarizing we have

$$
\bar{L}^{p}[\beta]=\left[\beta_{p r}\right] \in H^{q-p}\left(\mathcal{P}^{*}\left(M^{2 n}\right), d_{l}^{+}\right)=E_{l+p, 1}^{p, q} .
$$

4. Substituting for $0 \leq p=q \leq n-1$ in the long exact sequence (4.12), we get the left end of (4.12)

$$
0 \rightarrow H_{l}^{0}\left(M^{2 n}\right) \rightarrow E_{l+p, 1}^{p, p} \rightarrow 0 \rightarrow H_{l}^{1}\left(M^{2 n}\right) \rightarrow \cdots .
$$

From (4.7) and (4.15) we get for $0 \leq p \leq n-1$

$$
E_{l+p, 1}^{p, p}=H^{0,+}\left(\mathcal{P}^{*}\left(M^{2 n}\right)\right)=H_{l}^{0}\left(M^{2 n}\right)
$$

Let us prolong the exact sequence (4.12) for $q=n$, using the ideas in [7, $\S \mathrm{III}]$. For $0 \leq k \leq n$ we set

$$
C_{l}^{k}:=\frac{\operatorname{ker} d_{l}^{-} \cap \Omega^{k}\left(M^{2 n}\right)}{d_{l}\left(\Omega^{k-1}\left(M^{2 n}\right)\right)}
$$

Proposition 4.5. The long exact sequence (4.12) can be extended as follows (4.17)

$$
E_{l+p, 1}^{p, n-1} \stackrel{\delta_{p, n-1}}{\rightarrow} H_{l-1}^{n-(p+2)}\left(M^{2 n}\right) \stackrel{[L]}{\rightarrow} C_{l}^{n-p} \stackrel{\left[L^{p}\right]}{\rightarrow} E_{l+p, 1}^{p, n} \stackrel{\delta_{p, n}}{\rightarrow} C_{l-1}^{n-(p+1)} \stackrel{\left[L^{p+1}\right]}{\rightarrow} H_{l+p}^{n+p+1}\left(M^{2 n}\right) \rightarrow 0,
$$

where $0 \leq p \leq n-1$ and the operators $[L],\left[L^{p}\right]$ and $\left[L^{p+1}\right]$ will be defined in the proof below. 
Proof. First we define $[L]$ and prove the exactness at $H_{l-1}^{n-(p+2)}\left(M^{2 n}\right)$. Denote by $\Pi: H_{l}^{n-p}\left(M^{2 n}\right) \rightarrow C_{l}^{n-p}$ the natural embedding of the quotient spaces

$$
\frac{\operatorname{ker} d_{l} \cap \Omega^{n-p}\left(M^{2 n}\right)}{d_{l}\left(\Omega^{n-p-1}\left(M^{2 n}\right)\right)} \rightarrow \frac{\operatorname{ker} d_{l}^{-} \cap \Omega^{n-p}\left(M^{2 n}\right)}{d_{l}\left(\Omega^{n-p-1}\left(M^{2 n}\right)\right)} .
$$

Set $[L]:=\Pi \circ \bar{L}$, where $\bar{L}: H_{l-1}^{n-(p+2)}\left(M^{2 n}\right) \rightarrow H_{l}^{n-p}\left(M^{2 n}\right)$ is induced by $L$. By Theorem 4.3 we have $\operatorname{Im} \delta_{p, n-1}=\operatorname{ker} \bar{L}$. Since $\Pi$ is an embedding, the last equality implies $\operatorname{ker}[L]=\operatorname{ker} \bar{L}$. This proves the required exactness at $H_{l-1}^{n-(p+2)}\left(M^{2 n}\right)$.

Now we define $\left[L^{p}\right]$ and show the exactness at $C_{l}^{n-p}$. Assume that $\alpha=\alpha_{p r}+$ $L \tilde{\alpha} \in \Omega^{n-p}\left(M^{2 n}\right)$ is a representative of $[\alpha] \in C_{l}^{n-p}$, i.e. $d_{l}^{-}(\alpha)=0$, or equivalently $d_{l} \alpha=d_{l}^{+} \alpha$. We set

$$
\left[L^{p}\right](\alpha):=\left[\alpha_{p r}\right] \in \frac{\mathcal{P}^{n-p}\left(M^{2 n}\right)}{d_{l}^{+}\left(\mathcal{P}^{n-p-1}\left(M^{2 n}\right)\right)}=E_{l+p, 1}^{p, n} .
$$

Clearly the map $\left[L^{p}\right]$ is well-defined, since $\Pi_{p r} d_{l}(\gamma)=d_{l}^{+} \Pi_{p r}(\gamma)$. Now assume that $[\alpha] \in \operatorname{ker}\left[L^{p}\right]$, so by $(7.6)$

$$
\Pi_{p r} \alpha=d_{l}^{+} \gamma \text { for some } \gamma \in \mathcal{P}^{n-p-1}\left(M^{2 n}\right) .
$$

Using the property $d_{l} \alpha=d_{l}^{+} \alpha$ we obtain from (4.19) $d_{l} \alpha=0$. Now we write

$$
\alpha=\alpha_{p r}+L \tilde{\alpha}=d_{l}^{+} \gamma+L \tilde{\alpha}=d_{l} \gamma+L \beta,
$$

where $\beta=d_{l}^{-} \gamma+\tilde{\alpha}$. Since $d_{l} \alpha=0$, using (4.20) we get $d_{l} L \beta=L d_{l-1} \beta=0$. Applying Lemma 3.4 to $d_{l-1} \beta \in \Omega^{n-p-1}\left(M^{2 n}\right)$, we obtain $d_{l-1} \beta=0$. This implies $[\alpha]=[L]([\beta]) \in \operatorname{Im}[L]$, and the required exactness.

Next we define the operator $\delta_{p, n}: E_{l+p, 1}^{p, n} \rightarrow C_{l-1}^{n-(p+1)}$ as follows

$$
\delta_{p, n}(\alpha):=\left[d_{l}^{-} \tilde{\alpha}\right] \in C_{l-1}^{n-(p+1)},
$$

where $\tilde{\alpha} \in \mathcal{P}^{n-p}\left(M^{2 n}\right)$ is a representative of $\alpha \in E_{l+p, 1}^{p, n}=$ $\mathcal{P}^{n-p}\left(M^{2 n}\right) / d_{l}^{+}\left(\mathcal{P}^{n-p-1}\left(M^{2 n}\right)\right) . \quad$ Clearly $\delta_{p, n}(\alpha) \in C_{l-1}^{n-p-1}$, since by (3.14) $d_{l-1}\left(d_{l}^{-} \tilde{\alpha}\right)=d_{l-1}^{+} d_{l}^{-} \tilde{\alpha}$. The map $\delta_{p, n}$ is well-defined, since for any $\gamma \in \mathcal{P}^{n-p-1}\left(M^{2 n}\right)$ using (3.14) and (3.15) we get

$$
\left[d_{l}^{-} d_{l}^{+} \gamma\right]=-\left[d_{l-1}^{+} d_{l}^{-} \gamma\right]=-\left[d_{l-1}\left(d_{l}^{-} \gamma\right)\right]=0 \in C_{l-1}^{n-p-1}
$$

Now assume that $\alpha \in \operatorname{ker} \delta_{p, n}$ and $\tilde{\alpha} \in \mathcal{P}^{n-p}\left(M^{2 n}\right)$ is its representative. By (4.21) $d_{l}^{-} \tilde{\alpha}=d_{l-1} \beta$ for some $\beta \in \Omega^{n-p-2}\left(M^{2 n}\right)$. It follows

$$
d_{l}^{+} \tilde{\alpha}=d_{l} \tilde{\alpha}-L d_{l-1} \beta=d_{l}(\tilde{\alpha}-L \beta) .
$$

Since $\tilde{\alpha}$ is primitive, and $d_{l}^{+}\left(\mathcal{L}^{s, r}\right) \subset \mathcal{L}^{s, r+1}$, we get

$$
d_{l}^{+} \tilde{\alpha}=d_{l}^{+}(\tilde{\alpha}-L \beta)
$$

Clearly, (4.23) and (4.24) imply $[\tilde{\alpha}-L \beta] \in C_{l}^{n-p}$, and by (7.6) $\alpha=\left[L^{p}\right]([\tilde{\alpha}-L \beta])$. This yields the exactness at $E_{l+p, 1}^{p, n}$. 
Let us define $\left[L^{p+1}\right]$ and show the exactness at $C_{l-1}^{n-(p+1)}$. For $\alpha \in C_{l-1}^{n-p-1}$ we set

$$
\left[L^{p+1}\right](\alpha):=\left[L^{p+1} \tilde{\alpha}\right] \in H_{l+p}^{n+p+1}\left(M^{2 n}\right),
$$

where $\tilde{\alpha} \in \Omega^{n-p-1}\left(M^{2 n}\right)$ is a representative of $\alpha$. Note that $d_{l+p} L^{p+1} \tilde{\alpha}=$ $L^{p+1} d_{l-1} \tilde{\alpha}=0$, so $\left[L^{p+1}\right](\alpha) \in H_{l+p}^{n+p+1}\left(M^{2 n}\right)$. The same formula shows that our map $\left[L^{p+1}\right]$ does not depend on the choice of a representative $\tilde{\alpha}$ of $\alpha$. Now assume that $\alpha \in \operatorname{ker}\left[L^{p+1}\right]$. Then $L^{p+1} \tilde{\alpha}=d_{l+p} \beta$ for some $\beta \in \Omega^{n+p}\left(M^{2 n}\right)$. Using the Lefschetz decomposition for $\beta$ we write

$$
\beta=L^{p}\left(\beta_{0}+\sum_{k=1}^{\left[\frac{n+p}{2}\right]} L^{k} \beta_{k}\right), \beta_{i} \in \mathcal{P}^{*}\left(M^{2 n}\right) .
$$

It follows that

$$
L^{p+1} \tilde{\alpha}=d_{l+p} \beta=L^{p}\left(d_{l}^{+} \beta_{0}+L\left(d_{l}^{-} \beta_{0}+d_{l-1}\left(\beta_{1}+L \beta_{2}+\cdots\right)\right)\right) .
$$

By Corollary 3.4.1 $L^{p+1}: \Omega^{n-p-1}\left(M^{2 n}\right) \rightarrow \Omega^{n+p+1}\left(M^{2 n}\right)$ is an isomorphism, hence we get from (4.26)

$$
\tilde{\alpha}=d_{l}^{-} \beta_{p}+d_{l-1}\left(\beta_{p+1}+L \beta_{p+1}+\cdots\right) .
$$

Combining (4.27) with (4.21) we get $\alpha \in \operatorname{Im} \delta_{p, n}$. This proves the required exactness.

Finally we show that $\left[L^{p+1}\right]$ is surjective. Assume that $\beta \in \Omega^{n+p+1}\left(M^{2 n}\right)$ is a representative of $[\beta] \in H_{l+p+1}^{n+p+1}\left(M^{2 n}\right)$. Using the Lefschetz decomposition we write $\beta=L^{p+1}(\tilde{\beta}), \tilde{\beta} \in \Omega^{n-p-1}\left(M^{2 n}\right)$. Note that $L^{p+2} d_{l}^{-} \tilde{\beta}=L d_{l+p}^{-} \beta=0$, since $d_{l+p} \beta=0$. Since $L^{p+2}: \Omega^{n-p-2}\left(M^{2 n}\right) \rightarrow \Omega^{n+p+2}\left(M^{2 n}\right)$ is an isomorphism, we get $d_{l}^{-} \tilde{\beta}=0$. Hence $[\tilde{\beta}] \in C_{l}^{n-p-1}$ and $[\beta]=\left[L^{p+1}\right]([\tilde{\beta}])$. This completes the proof of Proposition 4.5.

Theorem 4.6. (cf. [8, Osservazione 18]) The spectral sequences $E_{k, r}^{p, q}$ on $\left(M^{2 n}, \omega, \theta\right)$ and on $\left(M^{2 n}, \omega^{\prime}, \theta^{\prime}\right)$ are isomorphic, if $\omega$ and $\omega^{\prime}$ are conformal equivalent. Furthermore, the $E_{k, 1}^{*, *}$-terms of the spectral sequences on $(M, \omega, \theta)$ and $\left(M, \omega^{\prime}, \theta^{\prime}\right)$ are isomorphic, if $\omega^{\prime}=\omega+d_{\theta} \rho$ for some $\rho \in \Omega^{1}\left(M^{2 n}\right)$.

Proof. If $\omega^{\prime}= \pm e^{f} \omega$, then $d_{\theta} \omega^{\prime}=d f \wedge \omega^{\prime}$. Hence

$$
\left(d_{\theta}-d f \wedge\right) \omega^{\prime}=0 .
$$

Since $L: \Omega^{1}\left(M^{2 n}\right) \rightarrow \Omega^{3}\left(M^{2 n}\right)$ is injective, (4.28) implies that

$$
d_{\theta^{\prime}}=d_{\theta}-d f \wedge .
$$

It follows

$$
d_{k \theta^{\prime}}=d_{k \theta}-k \cdot d f \wedge .
$$

Hence the map $I_{f}: \alpha \mapsto e^{k f} \alpha$ is an isomorphism between complexes $\left(F^{*} K^{*}, d_{k \theta}\right)$ and $\left(F^{*} K^{*}, d_{k \theta^{\prime}}\right)$, since

$$
d_{k \theta^{\prime}}\left(e^{k f} \alpha\right)=d_{k \theta}\left(e^{k f} \alpha\right)+(-k \cdot d f) \wedge e^{k \cdot f} \alpha=e^{k \cdot f}\left(d_{k \theta} \alpha\right) .
$$


It follows that the resulting terms $E_{k, 0}^{p, q}$ are also conformal equivalent. Moreover, the map $I_{f}$ induces an isomorphism $I_{f}^{0}$ between complexes

$$
I_{f}^{0}:\left(E_{k, 0}^{p, q}, d_{k, 0}\right) \rightarrow\left(E_{k, 0}^{p, q}, d_{k, 0}^{\prime}\right),[\alpha] \mapsto\left[e^{k f} \alpha\right] .
$$

Inductively, this proves the first assertion of Theorem 4.6.

Now we assume that $\omega^{\prime}=\omega+d_{\theta} \rho$. Then $d_{\theta} \omega^{\prime}=0$. Using the injectivity of $L: \Omega^{1}\left(M^{2 n}\right) \rightarrow \Omega^{3}\left(M^{2 n}\right)$ we conclude that the Lee form of $\omega^{\prime}$ is equal to the Lee form $\theta$ of $\omega$. Denote by $L^{\prime}$ the wedge product with $\omega^{\prime}$, and by $\left[L^{\prime}\right]$ the induced operator on $H_{l}^{*}\left(M^{2 n}\right)$. Using $\omega^{\prime}-\omega=d_{\theta} \rho$ and applying (2.6), which implies that the wedge product with $d_{\theta} \rho$ maps $H_{l}^{k}\left(M^{2 n}\right)$ to zero, we conclude that the operators $L$ and $L^{\prime}$ induce the same map $H_{l}^{k}\left(M^{2 n}\right) \rightarrow H_{l+1}^{k+2}\left(M^{2 n}\right)$.

To prove the second assertion of Theorem 4.6 we use the following version of Five Lemma, whose proof is obvious and hence omitted.

Lemma 4.7. Assume that the following diagram of vector spaces $A_{i}, B_{i}$ over a field $\mathbb{F}$ is commutative. If the rows are exact and $A_{1} \rightarrow B_{1}, A_{2} \rightarrow B_{2}, A_{4} \rightarrow B_{4}$, $A_{5} \rightarrow B_{5}$ are isomorphisms, then there is an isomorphism from $A_{3}$ to $B_{3}$, which also commutes with the other arrows.

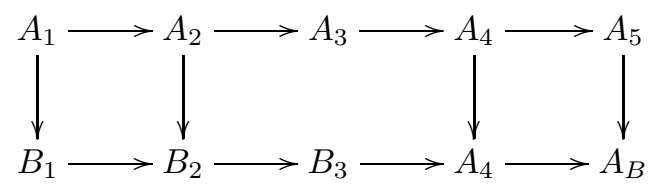

The second assertion of Theorem 4.6 for $E_{k, 1}^{p, q}$ follows immediately from the long exact sequence (4.12) and the formula (4.31), if $0 \leq p \leq q \leq n-1$, taking into account Lemma 4.1.

To examine the term $E_{k, 1}^{p, n}, 0 \leq p \leq n-1$, we need the following

LEMma 4.8. Assume that $\omega^{\prime}=\omega+d_{\theta} \rho$. For $0 \leq p \leq n$ there are linear maps $B_{l}^{n-p}: C_{l}^{n-p}(\omega) \rightarrow C_{l}^{n-p}\left(\omega^{\prime}\right)$ such that the following two diagrams are commutative. (The symbol I denotes the identity mapping. The other mapping are defined in the proof.)

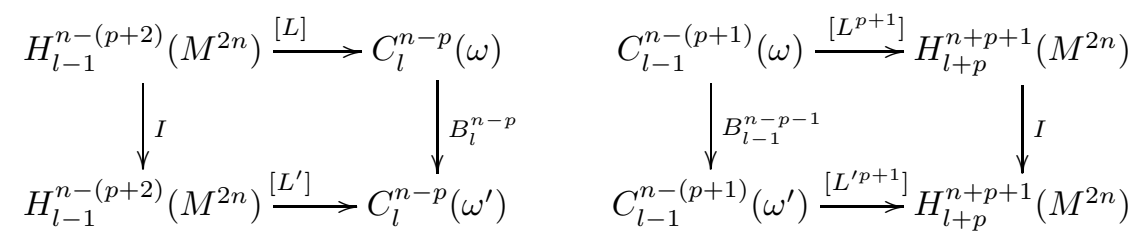

Proof. Let us define first a linear mapping

$$
\tilde{B}_{l}^{n-p}: \operatorname{ker} d_{l}^{-}(\omega) \cap \Omega^{n-p}\left(M^{2 n}\right) \rightarrow \Omega^{n-p}\left(M^{2 n}\right) .
$$

Let $\eta \in \operatorname{ker} d_{l}^{-} \cap \Omega^{n-p}\left(M^{2 n}\right)$. This means that $d_{l} \eta=d_{l}^{+} \eta$ or equivalently that $d_{l} \eta$ is primitive. The last assertion is again equivalent to the equality $\omega^{p} \wedge d_{l} \eta=0$. Since $\left(L^{\prime}\right)^{p}: \Omega^{n-p}\left(M^{2 n}\right) \rightarrow \Omega^{n+p}\left(M^{2 n}\right)$ is an isomorphism, there is a unique $\eta^{\prime} \in$ $\Omega^{n-p}\left(M^{2 n}\right)$ such that

$$
\sum_{i=1}^{p}\left(\begin{array}{c}
p \\
i
\end{array}\right) \rho \wedge\left(d_{\theta} \rho\right)^{i-1} \wedge \omega^{p-i} \wedge d_{l} \eta=\omega^{\prime p} \wedge \eta^{\prime} .
$$


Now we define $\tilde{B}_{l}^{n-p}$ by

$$
\tilde{B}_{l}^{n-p} \eta:=\eta-\eta^{\prime}
$$

We shall now prove that the element $d_{l}\left(\eta-\eta^{\prime}\right)$ is $\omega^{\prime}$-primitive.

$$
\begin{gathered}
\omega^{\prime p} \wedge d_{l}\left(\eta-\eta^{\prime}\right)=-\omega^{\prime p} \wedge d_{l} \eta^{\prime}+\left(\omega+d_{1} \rho\right)^{p} \wedge d_{l} \eta= \\
=-d_{p+l}\left(\omega^{\prime p} \wedge \eta^{\prime}\right)+\omega^{p} \wedge d_{l} \eta+\sum_{i=1}^{p}\left(\begin{array}{c}
p \\
i
\end{array}\right)\left(d_{1} \rho\right)^{i} \wedge \omega^{p-i} \wedge d_{l} \eta= \\
=-d_{p+l}\left(\omega^{\prime p} \wedge \eta^{\prime}\right)+d_{p+l}\left[\sum_{i=1}^{p}\left(\begin{array}{c}
p \\
i
\end{array}\right) \rho \wedge\left(d_{1} \rho\right)^{i-1} \wedge \omega^{p-i} \wedge d_{l} \eta\right]= \\
=d_{p+l}\left[-\omega^{\prime p} \wedge \eta^{\prime}+\sum_{i=1}^{p}\left(\begin{array}{c}
p \\
i
\end{array}\right) \rho \wedge\left(d_{1} \rho\right)^{i-1} \wedge \omega^{p-i} \wedge d_{l} \eta\right]=0 .
\end{gathered}
$$

In other words we have proved that $\tilde{B}_{l}^{n-p}$ maps $\operatorname{ker} d_{l}^{-} \cap \Omega^{n-p}\left(M^{2 n}\right)$ into $\operatorname{ker}\left(d^{\prime}\right)_{l}^{-} \cap$ $\Omega^{n-p}\left(M^{2 n}\right)$, where $\left(d^{\prime}\right)_{l}^{-}$is defined via the Lefschetz decomposition corresponding to $\omega^{\prime}$.

Let us take now an element $\eta \in d_{l} \Omega^{n-p-1}\left(M^{2 n}\right)$, i. e. $\eta=d_{l} \gamma$, where $\gamma \in$ $\Omega^{n-p-1}\left(M^{2 n}\right)$. Then $d_{l} \eta=0$ and we have $\omega^{p} \wedge \eta^{\prime}=0$, which implies $\eta^{\prime}=0$. We thus get $\tilde{B}_{l}^{n-p} \eta=\eta$. Consequently we have proved that $\tilde{B}_{l}^{n-p}$ maps $d_{l}\left(\Omega^{n-p-1}\left(M^{2 n}\right)\right)$ into itself. Now it is obvious that $\tilde{B}_{l}^{n-p}$ induces a linear mapping

$$
B_{l}^{n-p}: C_{l}^{n-p}(\omega) \rightarrow C_{l}^{n-p}\left(\omega^{\prime}\right) .
$$

Next we shall investigate the first diagram. First we define the mapping $[L]$. If $[\beta] \in H_{l-1}^{n-(p+2)}\left(M^{2 n}\right)$, then we have an element $\beta \in \Omega^{n-(p+2)}\left(M^{2 n}\right)$ such that $d_{l-1} \beta=0$. Let us set

$$
[L][\beta]:=[\omega \wedge \beta] .
$$

It is easy to see that this definition depends only on the cohomology class $[\beta] \in$ $H_{l-1}^{n-p-2}\left(M^{2 n}\right)$. Namely, if $\tilde{\beta}=\beta+d_{l-1} \gamma$, then

$$
\omega \wedge\left(\beta+d_{l-1} \gamma\right)=\omega \wedge \beta+d_{l}(\omega \wedge \gamma),
$$

which shows that $[L][\tilde{\beta}]=[L][\beta]$. Similarly we define $\left[L^{\prime}\right]$. Let us take $[\beta] \in$ $H_{l-1}^{n-(p+2)}\left(M^{2 n}\right)$. Then $d_{l-1} \beta=0$, and we have

$$
\begin{gathered}
\sum_{i=1}^{p}\left(\begin{array}{c}
p \\
i
\end{array}\right) \rho \wedge\left(d_{1} \rho\right)^{i-1} \wedge \omega^{p-i} \wedge d_{l}(\omega \wedge \beta)= \\
=\sum_{i=1}^{p}\left(\begin{array}{c}
p \\
i
\end{array}\right) \rho \wedge\left(d_{1} \rho\right)^{i-1} \wedge \omega^{p-i} \wedge\left(d_{1} \omega \wedge \beta+\omega \wedge d_{l-1} \beta\right)=0 .
\end{gathered}
$$

We have $0=\omega^{\prime p} \wedge \eta^{\prime}$, which implies $\eta^{\prime}=0$. Thus we get $B_{l}^{n-p}[L][\beta]=B_{l}^{n-p}[\omega \wedge \beta]=$ $[\omega \wedge \beta-0]=[\omega \wedge \beta]$. On the other hand we compute

$$
\begin{gathered}
\left.\left[L^{\prime}\right][\beta]=\left[\omega^{\prime} \wedge \beta\right]=\left[\left(\omega+d_{1} \rho\right) \wedge \beta\right)\right]=[\omega \wedge \beta]+\left[d_{1} \rho \wedge \beta+\rho \wedge d_{l-1} \beta\right]= \\
=[\omega \wedge \beta]+\left[d_{l}(\rho \wedge \beta)\right]=[\omega \wedge \beta] .
\end{gathered}
$$


We have thus shown that that the first diagram is commutative.

We continue now with the second diagram. Again we define first the mapping $\left[L^{p+1}\right]$. For $[\eta] \in C_{l-1}^{n-(p+1)}(\omega)$ there is a representative $\eta \in \Omega_{l-1}^{n-(p+1)}\left(M^{2 n}\right)$ such that $d_{l-1}^{-} \eta=0$. This means that $d_{l-1}=d_{l-1}^{+} \eta$. We compute

$$
\begin{gathered}
d_{l+p}\left(\omega^{p+1} \wedge \eta\right)=d_{(p+1)+(l-1)}\left(\omega^{p+1} \wedge \eta\right)=d_{p+1}\left(\omega^{p+1}\right) \wedge \eta+\omega^{p+1} \wedge d_{l-1} \eta= \\
=\omega^{p+1} \wedge d_{l-1}^{+} \eta=0 .
\end{gathered}
$$

The last term vanishes because the form $d_{l-1}^{+} \eta$ is primitive. Finally let us suppose that $\eta=d_{l-1} \gamma$. Then we have

$$
\omega^{p+1} \wedge d_{l-1} \gamma=d_{p+1}\left(\omega^{p+1}\right) \wedge \gamma+\omega^{p+1} \wedge d_{l-1} \gamma=d_{(p+1)+(l-1)}\left(\omega^{p+1} \wedge \gamma\right) .
$$

This shows that we can define $\left[L^{p+1}\right]$ by the formula

$$
\left[L^{p+1}\right][\eta]:=\left[\omega^{p+1} \wedge \eta\right] .
$$

Now we are going to prove the commutativity of the second diagram. For $[\eta] \in$ $C_{l-1}^{n-(p+1)}(\omega)$ we have $B_{l-1}^{n-p-1}[\eta]=\left[\eta-\eta^{\prime}\right]$, where $\eta^{\prime}$ is uniquely determined by the equality

$$
\omega^{\prime p+1} \wedge \eta^{\prime}=\sum_{i=1}^{p+1}\left(\begin{array}{c}
p+1 \\
i
\end{array}\right) \rho \wedge\left(d_{1} \rho\right)^{i-1} \wedge \omega^{p+1-i} \wedge d_{l-1} \eta .
$$

Further we have $\left[L^{\prime}\right] B_{l-1}^{n-p-1}[\eta]=\left[\omega^{\prime} \wedge\left(\eta-\eta^{\prime}\right)\right]$. Now let us compute

$$
\begin{gathered}
\omega^{\prime p+1} \wedge\left(\eta-\eta^{\prime}\right)-\omega^{p+1} \wedge \eta=\omega^{\prime p+1} \wedge \eta-\omega^{\prime p+1} \wedge \eta^{\prime}-\omega^{p+1} \wedge \eta= \\
\left.=\left(\omega+d_{1} \rho\right)\right)^{p+1} \wedge \eta-\sum_{i=1}^{p+1}\left(\begin{array}{c}
p+1 \\
i
\end{array}\right) \rho \wedge\left(d_{1} \rho\right)^{i-1} \wedge \omega^{p+1-i} \wedge d_{l-1} \eta-\omega^{p+1} \wedge \eta= \\
=\sum_{i=1}^{p+1}\left(\begin{array}{c}
p+1 \\
i
\end{array}\right)\left(d_{1} \rho\right)^{i} \wedge \omega^{p+1-i} \wedge \eta-\sum_{i=1}^{p+1}\left(\begin{array}{c}
p+1 \\
i
\end{array}\right) \rho \wedge\left(d_{1} \rho\right)^{i-1} \wedge \omega^{p+1-i} \wedge d_{l-1} \eta= \\
=d_{p+1}\left(\sum_{i=1}^{p+1}\left(\begin{array}{c}
p+1 \\
i
\end{array}\right) \rho \wedge\left(d_{1} \rho\right)^{i-1} \wedge \omega^{p+1-i}\right) \wedge \eta \\
-\left(\sum_{i=1}^{p+1}\left(\begin{array}{c}
p+1 \\
i
\end{array}\right) \rho \wedge\left(d_{1} \rho\right)^{i-1} \wedge \omega^{p+1-i}\right) \wedge d_{l-1} \eta= \\
\left.=d_{(p+1)+(l-1)}\left(\begin{array}{c}
p+1 \\
\sum_{i=1}^{p+1}
\end{array}\right) \rho \wedge\left(d_{1} \rho\right)^{i-1} \wedge \omega^{p+1-i} \wedge \eta\right) .
\end{gathered}
$$

We have thus proved that $\left[L^{\prime p+1}\right] B_{l-1}^{n-p-1}[\eta]=\left[L^{p+1}\right][\eta]$.

Clearly the second assertion of Proposition 4.6 for $E_{k, 1}^{p, n}, 0 \leq p \leq n-1$, follows from Proposition 4.5, Lemma 4.7, and Lemma 4.8. Combining with (4.8), which implies that $E_{k, 1}^{n, n}=C^{\infty}\left(M^{2 n}\right)$, we obtain the second assertion of Proposition 4.6. This completes the proof of Theorem 4.6.

REMARK 4.9. In [11, Example 7.1] the authors construct an example of a compact 6-dimensional nilmanifold $M^{6}$ equipped with a family of symplectic forms $\omega_{t}, t \in[0,1]$, 
with varying cohomology classes $\left[\omega_{t}\right] \in H^{2}\left(M^{6}, \mathbb{R}\right)$. They showed that the coeffective cohomology groups associated to $\omega_{1}$ and $\omega_{2}$ have different Betti number $b_{4}$. It follows that, using [29, Lemma 2.7, Proposition 3.5, part II], see also Remark 3.16 .1 above, the $E_{1}$-terms of the associated spectral sequences for $\omega_{0}$ and $\omega_{1}$ are different.

5. The stabilization of the spectral sequences. In this section we prove that the spectral sequences $\left\{E_{l, r}^{p, q}\right\}$ on l.c.s.manifolds $\left(M^{2 n}, \omega, \theta\right)$ converge to the Lichnerowicz-Novikov cohomology $H_{l}^{*}\left(M^{2 n}\right)$ at the second term $E_{l, 2}^{*, *}$, or at the $t$ term $E_{l, t}^{*, *}$ under some cohomological conditions posed on $\omega$ (Theorems 5.2, 5.8, 5.13). As a consequence, we obtain a relation between the primitive cohomology groups and the de Rham cohomology groups of $\left(M^{2 n}, \omega\right)$, if $\left(M^{2 n}, \omega\right)$ is a compact symplectic manifold. This gives an answer to a question posed by Tseng and Yau in [29], see Remark 5.18.

First we prove the following simple property of the second terms $E_{l, 2}^{*, *}$, which will be used later in the proof of Theorem 5.13.

Proposition 5.1. (cf. [8, Proposizione 19]) Assume that $1 \leq p \leq q \leq n-1$. Then $E_{l, 2}^{p, q}=E_{l, 2}^{p-1, q-1}$.

Proof. Let $\alpha \in E_{l+p, 1}^{p, q}=H^{q-p}\left(\mathcal{P}^{*}\left(M^{2 n}\right), d_{l}^{+}\right)$and $\tilde{\alpha} \in \mathcal{P}^{q-p}\left(M^{2 n}\right)$ its representative as in (4.7). The differential $d_{l+p, 1}: E_{l+p, 1}^{p, q} \rightarrow E_{l+p, 1}^{p+1, q}$ is defined by

$$
d_{l+p, 1}(\alpha):=\left[d_{l+p} L^{p} \tilde{\alpha}\right]=\left[L^{p} d_{l} \tilde{\alpha}\right] \in E_{l+p, 1}^{p+1, q} .
$$

Using $d_{l+p}=d_{l+p}^{+}+L d_{l+p}^{-}$and taking into account $d_{l}^{+} \tilde{\alpha}=0$, we observe that $\left[L^{p} d_{l} \tilde{\alpha}\right] \in$ $E_{l+p, 1}^{p+1, q}$ has a representative $d_{l}^{-}(\tilde{\alpha}) \in \mathcal{P}^{q-p-1}\left(M^{2 n}\right)$ in $H^{q-p-1}\left(\mathcal{P}^{*}\left(M^{2 n}\right), d_{l-1}^{+}\right)=$ $E_{l+p, 1}^{p+1, q}$, if $0 \leq p \leq q \leq n$. Equivalently, using (4.7), we rewrite $d_{l+p, 1}$ for $0 \leq p \leq q \leq n$ as follows

$$
d_{l+p, 1}: H^{q-p}\left(\mathcal{P}^{*}\left(M^{2 n}\right), d_{l}^{+}\right) \rightarrow H^{q-p-1}\left(\mathcal{P}^{*}\left(M^{2 n}\right), d_{l-1}^{+}\right),[\tilde{\alpha}] \mapsto\left[d_{l}^{-} \tilde{\alpha}\right] .
$$

Clearly Proposition 5.1 follows from (4.7) and the formula (5.1), (5.2).

Now assume that $\omega=d_{k} \tau$ for some $k \in \mathbb{Z}$ and $\tau \in \Omega^{1}\left(M^{2 n}\right)$. Since $d_{1} \omega=d_{k} \omega=$ 0 , it follows that $(k-1) \theta \wedge \omega=0$. Since $L$ is injective, we get $k=1$. The following theorem is a generalization of [7, Theorem 2] for the symplectic case $\theta=0$.

Theorem 5.2. (cf. [7, Theorem 2]) Assume that $\omega=d_{1} \tau$. Then $E_{l, 2}^{p, q}=0$, if $1 \leq p \leq q \leq n-1$. If $0 \leq q \leq n$, then $E_{l, 2}^{0, q}=H_{l}^{q}\left(M^{2 n}\right)$. If $0 \leq p \leq n$ then $E_{l+p, 2}^{p, n}=H_{l+p}^{n+p}\left(M^{2 n}\right)$. Thus the spectral sequence $\left\{E_{l, r}^{p, q}, d_{l, r}\right\}$ stabilizes at the term $E_{l, 2}$.

Proof. Assume that $\omega=d_{1} \tau$. Then for any $d_{l-1}$-closed form $\alpha$ we have

$$
d_{1} \tau \wedge \alpha=d_{l}(\tau \wedge \alpha) .
$$

Hence the induced operator $\bar{L}$ in the exact sequence (4.12) satisfies

$$
\bar{L}\left(H_{l-1}^{q-(p+1)}\left(M^{2 n}\right)\right)=0 \in H_{l}^{q+1-p}\left(M^{2 n}\right) .
$$

The equality (5.4) and the exact sequence (4.12) lead to the following exact sequence for $0 \leq p \leq q \leq n-1$.

$$
0 \rightarrow H_{l}^{q-p}\left(M^{2 n}\right) \stackrel{\bar{L}^{p}}{\rightarrow} E_{l+p, 1}^{p, q} \stackrel{\delta_{p, q}}{\rightarrow} H_{l-1}^{q-(p+1)}\left(M^{2 n}\right) \rightarrow 0 .
$$


Using the isomorphism $E_{l+p, 1}^{p, q}=H^{q-p}\left(\mathcal{P}^{*}\left(M^{2 n}\right), d_{l}^{+}\right)$and the formulae (4.13) and (4.14) describing $\delta_{p, q}$ and $\bar{L}^{p}$, we rewrite the exact sequence (5.5) as follows

$$
0 \rightarrow H_{l}^{q-p}\left(M^{2 n}\right) \stackrel{\left[\Pi_{p r}\right]}{\rightarrow} H^{q-p}\left(\mathcal{P}^{*}\left(M^{2 n}\right), d_{l}^{+}\right) \stackrel{\left[d_{l}^{-}\right]}{\rightarrow} H_{l-1}^{q-(p+1)}\left(M^{2 n}\right) \rightarrow 0 .
$$

The proof of the first and second assertion of Theorem 5.2 is based on our analysis of the long exact sequence of cohomology groups associated with the short exact sequence (5.6). By (5.2), for $0 \leq p \leq q \leq n-1$, the differential $d_{l+p, 1}: E_{l+p, 1}^{p, q} \rightarrow$ $E_{l+p, 1}^{p+1, q}$ induces the following boundary operator

$$
\hat{d}_{l}^{-}: H^{q-p}\left(\mathcal{P}^{*}\left(M^{2 n}\right), d_{l}^{+}\right) \rightarrow H^{q-p-1}\left(\mathcal{P}^{*}\left(M^{2 n}\right), d_{l-1}^{+}\right),[\tilde{\alpha}] \mapsto\left[d_{l}^{-} \tilde{\alpha}\right],
$$

for $\tilde{\alpha} \in \mathcal{P}^{q-p}\left(M^{2 n}\right)$.

LEMMA 5.3. The short exact sequence (5.6) generates a short exact sequence of the following chain complexes for $1 \leq p \leq q \leq n-1$ :

$$
\begin{aligned}
0 \rightarrow\left(H_{l}^{q-p}\left(M^{2 n}\right), \tilde{d}_{l}:=\right. & 0) \stackrel{\left[\Pi_{p r}\right]}{\rightarrow}\left(H^{q-p}\left(\mathcal{P}^{*}\left(M^{2 n}\right), d_{l}^{+}\right), \hat{d}_{l}^{-}\right) \rightarrow \\
& \stackrel{\left[d_{l}^{-}\right]}{\rightarrow}\left(H_{l-1}^{q-(p+1)}\left(M^{2 n}\right), \tilde{d}_{l-1}:=0\right) \rightarrow 0 .
\end{aligned}
$$

Proof. It is useful to rewrite the sequence (5.8) of chain complexes as the following diagram

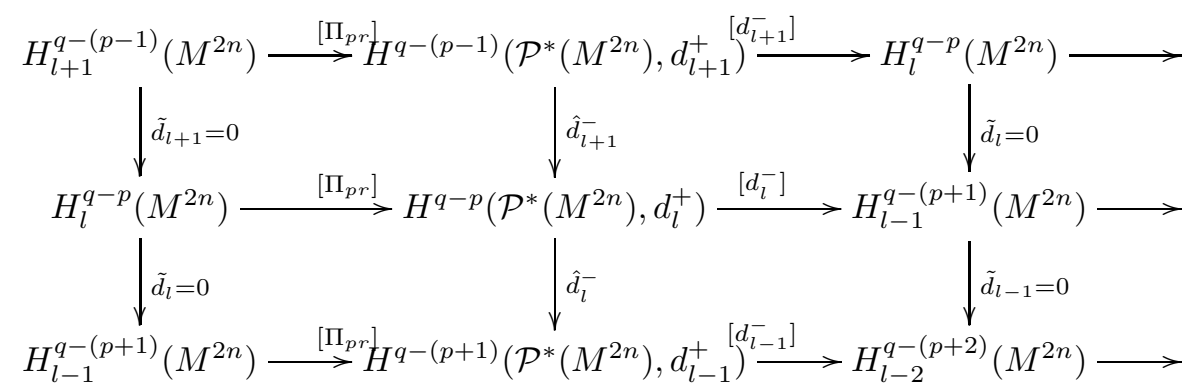

To prove Lemma 5.3, it suffices to show that the above diagram is commutative, or equivalently

$$
\begin{gathered}
\hat{d}_{l}^{-}\left[\Pi_{p r}\right]=\tilde{d}_{l}\left[\Pi_{p r}\right]=0, \\
{\left[d_{l-1}^{-}\right] \hat{d}_{l}^{-}=\tilde{d}_{l-1}\left[d_{l}^{-}\right]=0 .}
\end{gathered}
$$

Let $\alpha \in H_{l}^{q-p}\left(M^{2 n}\right)$ and $\tilde{\alpha} \in \Omega^{q-p}\left(M^{2 n}\right)$ its representative. Let $\tilde{\alpha}=\tilde{\alpha}_{p r}+L \tilde{\beta}_{p r}+$ $L^{2} \gamma$ be the Lefschetz decomposition of $\tilde{\alpha}$. Using $d_{l} \tilde{\alpha}=d_{l}^{+} \tilde{\alpha}=d_{l}^{+} \tilde{\alpha}_{p r}=0$ we obtain $L d_{l}^{-} \tilde{\alpha}_{p r}=L d_{l-1}^{+} \tilde{\beta}_{p r}+L^{2}\left(d_{l-1}^{-} \tilde{\beta}_{p r}+d_{l-2} \gamma\right)$. Hence

$$
0=L\left(d_{l}^{-} \tilde{\alpha}_{p r}+d_{l-1}^{+} \tilde{\beta}_{p r}\right)+L^{2}\left(d_{l-1}^{-} \tilde{\beta}_{p r}+d_{l-2} \gamma\right)
$$

which implies $d_{l}^{-} \tilde{\alpha}_{p r}+d_{l-1}^{+} \tilde{\beta}_{p r}=0$ thanks to the uniqueness of the second Lefschetz decomposition. It follows

$$
\hat{d}_{l}^{-}\left[\Pi_{p r}\right] \alpha=\hat{d}_{l}^{-}\left[\tilde{\alpha}_{p r}\right]=\left[d_{l}^{-} \tilde{\alpha}_{p r}\right]=-\left[d_{l-1}^{+} \tilde{\beta}_{p r}\right]=0 \in H^{q-(p+1)}\left(\mathcal{P}^{*}\left(M^{2 n}\right), d_{l-1}^{+}\right) .
$$


Let $\beta \in H^{q-p}\left(\mathcal{P}^{*}\left(M^{2 n}\right), d_{l}^{+}\right)$and $\tilde{\beta} \in \mathcal{P}^{q-p}\left(M^{2 n}\right)$ its representative. Then

$$
\left[d_{l-1}^{-}\right] \hat{d}_{l}^{-} \beta=\left[d_{l-1}^{-} d_{l}^{-} \tilde{\beta}\right]=0 \in H_{l-2}^{q-(p+2)}\left(M^{2 n}\right) .
$$

Clearly (5.21) and (5.22) follow from (5.23) and (5.12). This completes the proof of Lemma 5.3.

The short exact sequence (5.8) in Lemma 5.3 generates the following associated long exact sequence of the cohomology groups

$$
\rightarrow E_{l+p, 2}^{p-1, q} \rightarrow H_{l}^{q-p}\left(M^{2 n}\right) \stackrel{\delta}{\rightarrow} H_{l}^{q-p}\left(M^{2 n}\right) \rightarrow E_{l+p, 2}^{p, q} \rightarrow H_{l-1}^{q-(p+1)}\left(M^{2 n}\right) \stackrel{\delta}{\rightarrow}
$$

where $\delta$ is the connecting homomorphism.

Lemma 5.4. We have $\delta(x)=x$ for all $x \in H_{l-1}^{q-(p+1)}\left(M^{2 n}\right)$ and for all $1 \leq p \leq$ $n-1$.

Proof of Lemma 5.6. Let $x \in H_{l-1}^{q-(p+1)}\left(M^{2 n}\right)$. Using (5.8) we write $x=\left[d_{l}^{-}\right] y, y \in$ $H^{q-p}\left(\mathcal{P}^{*}\left(M^{2 n}\right), d_{l}^{+}\right)$. By definition of the connecting homomorphism we have $\delta x=$ $\left[\hat{d}_{l}^{-} y\right]=x$. This completes the proof of Lemma 5.4 .

Clearly Lemma 5.4 implies the first assertion of Theorem 5.2.

Now let us consider the case $p=0, q \leq n-1$. Then $E_{l, 1}^{-1, q}=0$. The previous short exact sequence (5.8) of chain complexes is now replaced by the new one, where the cohomology groups on the line containing $E_{l, 1}^{-1, q}$ left and right to $E_{l, 1}^{-1, q}$ are zero. Let us write the new short exact sequence explicitly as the following commutative diagram

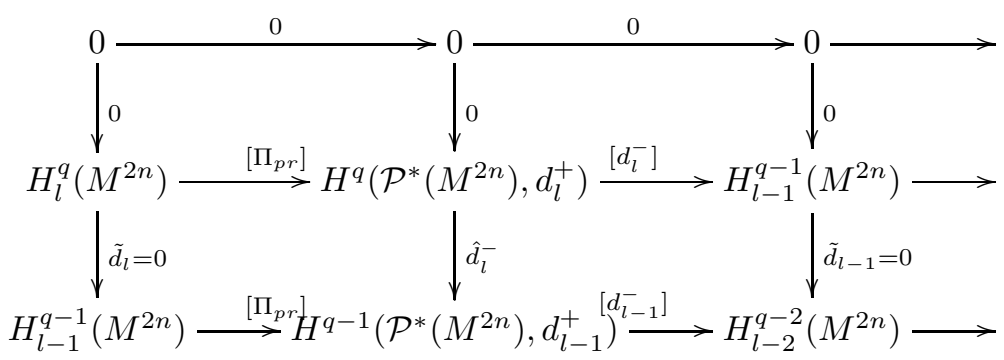

The resulting exact sequence of the cohomology groups now are

$$
0 \rightarrow H_{l}^{q}\left(M^{2 n}\right) \rightarrow E_{l, 2}^{0, q} \rightarrow H_{l-1}^{q-1}\left(M^{2 n}\right) \stackrel{\delta}{\rightarrow} H_{l-1}^{q-1}\left(M^{2 n}\right) \ldots
$$

Since $\delta=I d$, we obtain

$$
E_{l, 2}^{0, q}=H_{l}^{q}\left(M^{2 n}\right),
$$

which proves the second assertion of Theorem 5.2.

Next we compute $E_{l, 2}^{p, n}=E_{l, 1}^{p, n} / d_{l, 1}\left(E_{l, 1}^{p-1, n}\right)$ for $0 \leq p \leq n-1$. Since $\omega=d_{1} \tau$, the map $[L]: H_{l-1}^{n-(p+2)}\left(M^{2 n}\right) \rightarrow C_{l}^{n-p}$ sends $[\alpha]$ to $\left[d_{l}(\tau \wedge \tilde{\alpha})\right]=0 \in C_{l}^{n-p}$. Thus Proposition 4.5 implies that the following sequence is exact for $0 \leq p \leq n-1$

$$
0 \rightarrow C_{l}^{n-p} \stackrel{\left[L^{p}\right]}{\rightarrow} E_{l+p, 1}^{p, n} \stackrel{\delta_{p, n}}{\rightarrow} C_{l-1}^{n-(p+1)} \stackrel{\left[L^{p+1}\right]}{\rightarrow} H_{l+p}^{n+p+1}\left(M^{2 n}\right) \rightarrow 0 .
$$


Set for $-1 \leq p \leq n-1$

$$
T_{l-1}^{n-(p+1)}:=\operatorname{ker}\left[L^{p+1}\right]: C_{l-1}^{n-(p+1)} \rightarrow H_{l+p}^{n+p+1}\left(M^{2 n}\right) .
$$

Then we obtain from the exact sequence (5.15) the following short exact sequence

$$
0 \rightarrow C_{l}^{n-p} \stackrel{\left[L^{p}\right]}{\rightarrow} E_{l+p, 1}^{p, n} \stackrel{\delta_{p, n}}{\rightarrow} T_{l-1}^{n-(p+1)} \rightarrow 0 .
$$

Using the isomorphism $E_{l+p, 1}^{p, n}=\mathcal{P}^{n-p}\left(M^{2 n}\right) / d_{l}^{+}\left(\mathcal{P}^{n-p-1}\left(M^{2 n}\right)\right)$ and the formulas (4.18) and (4.21) describing $\left[L^{p}\right]$ and $\delta_{p, n}$ in the exact sequence (4.17) of Proposition 4.5 , we rewrite the short exact sequence (5.17) as follows

$$
0 \rightarrow C_{l}^{n-p} \stackrel{\left[\Pi_{p r}\right]}{\rightarrow} \frac{\mathcal{P}^{n-p}\left(M^{2 n}\right)}{d_{l}^{+}\left(\mathcal{P}^{n-p-1}\left(M^{2 n}\right)\right)} \stackrel{\left[d_{l}^{-}\right]}{\rightarrow} T_{l-1}^{n-(p+1)} \rightarrow 0 .
$$

Recall that the map $\left[\Pi_{p r}\right]$ is already defined in section 4 . It is the quotient map of the map $\Pi_{p r}:\left(\operatorname{ker} d_{l}^{-} \cap \Omega^{n-p}\left(M^{2 n}\right)\right) \rightarrow \mathcal{P}^{n-p}\left(M^{2 n}\right)$, see the explanation of (4.18).

Next recall that the map $\left[d_{l}^{-}\right]$is the quotient map of the map $d_{l}^{-}: \mathcal{P}^{n-p}\left(M^{2 n}\right) \rightarrow$ $\mathcal{P}^{n-p-1}\left(M^{2 n}\right) \cap \operatorname{ker} d_{l-1}^{-}$, see the explanation of (4.21). (We now explain why this map is also well-defined in (5.18). First we have $d_{l}^{-}\left(d_{l}^{+} \gamma\right)=d_{l-1}^{+} d_{l}^{-} \gamma=d_{l-1}\left(d_{l}^{-} \gamma\right)=$ $0 \in C_{l-1}^{n-(p+1)}$. Furthermore for $\alpha \in \mathcal{P}^{n-p}\left(M^{2 n}\right)$

$$
L^{p+1} d_{l}^{-}(\alpha)=L^{p}\left(L d_{l}^{-} \alpha\right)=L^{p} d_{l} \alpha=d_{l+p} L^{p} \alpha=0 \in H_{l+p}^{n+p+1}\left(M^{2 n}\right) .
$$

Hence

$$
\left[d_{l}^{-}\right]\left(\frac{\mathcal{P}^{n-p}\left(M^{2 n}\right)}{d_{l}^{+}\left(\mathcal{P}^{n-p-1}\left(M^{2 n}\right)\right)}\right) \subset T_{l-1}^{n-(p+1)}=\operatorname{ker}\left[L^{p+1}\right] .
$$

Thus $\left[d_{l}^{-}\right]$is well-defined.)

Note that the differential $d_{l+p, 1}: E_{l+p, 1}^{p, n} \rightarrow E_{l+p, 1}^{p+1, n}$ induces the following boundary operator

$$
\hat{d}_{l}^{-}: \frac{\mathcal{P}^{n-p}\left(M^{2 n}\right)}{d_{l}^{+}\left(\mathcal{P}^{n-p-1}\left(M^{2 n}\right)\right)} \rightarrow \frac{\mathcal{P}^{n-p-1}\left(M^{2 n}\right)}{d_{l-1}^{+}\left(\mathcal{P}^{n-p-2}\left(M^{2 n}\right)\right)},[\tilde{\alpha}] \mapsto\left[d_{l}^{-} \tilde{\alpha}\right],
$$

for $\tilde{\alpha} \in \mathcal{P}^{n-p}\left(M^{2 n}\right)$. The map $\hat{d}_{l}^{-}$is well-defined, since by (3.15) $d_{l}^{-} d_{l}^{+} \alpha=d_{l-1}^{+} d_{l}^{-} \alpha$ for $\alpha \in \mathcal{P}^{n-p-1}\left(M^{2 n}\right)$.

LEMMA 5.5. The short exact sequence (5.18) generates a short exact sequence of the following chain complexes

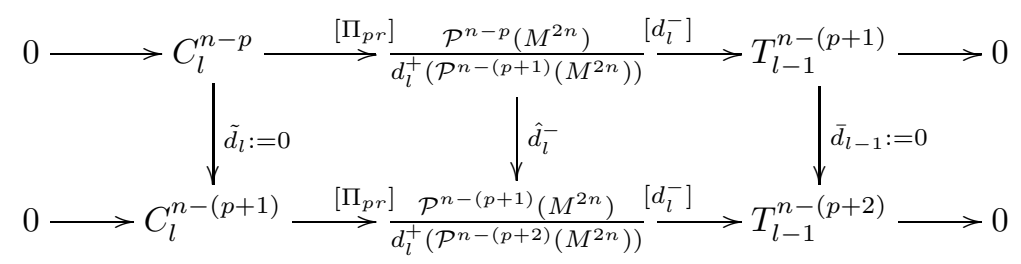

Proof of Lemma 5.5. It suffices to show that

$$
\begin{array}{r}
\hat{d}_{l}^{-}\left[\Pi_{p r}\right]=\tilde{d}_{l} \Pi_{p r}=0, \\
{\left[d_{l-1}^{-}\right] \hat{d}_{l}^{-}=\bar{d}_{l-1}\left[d_{l}^{-}\right]=0 .}
\end{array}
$$


Let $\alpha \in C_{l}^{n-p}$ and $\tilde{\alpha} \in \Omega^{n-p}\left(M^{2 n}\right)$ its representative. Let $\tilde{\alpha}=\tilde{\alpha}_{p r}+L \tilde{\beta}_{p r}+L^{2} \gamma$ be the Lefschetz decomposition of $\tilde{\alpha}$. Using $d_{l} \tilde{\alpha}=d_{l}^{+} \tilde{\alpha}$ we obtain $L d_{l}^{-} \tilde{\alpha}_{p r}=L d_{l-1}^{+} \tilde{\beta}_{p r}+$ $L^{2}\left(d_{l-1}^{-} \tilde{\beta}_{p r}+d_{l-2} \gamma\right)$. Hence

$$
\hat{d}_{l}^{-}\left[\Pi_{p r}\right] \alpha=\left[d_{l}^{-} \tilde{\alpha}_{p r}\right]=\left[d_{l-1}^{+} \tilde{\beta}_{p r}\right]=0 \in \frac{\mathcal{P}^{n-p-1}\left(M^{2 n}\right)}{d_{l-1}^{+}\left(\mathcal{P}^{n-p-2}\left(M^{2 n}\right)\right)} .
$$

Let $\beta \in\left(\mathcal{P}^{n-p}\left(M^{2 n}\right)\right) / d_{l}^{+}\left(\mathcal{P}^{n-p-1}\left(M^{2 n}\right)\right)$ and $\tilde{\beta} \in \mathcal{P}^{n-p}\left(M^{2 n}\right)$ its representative. Then

$$
\left[d_{l-1}^{-}\right] \hat{d}_{l}^{-}[\beta]=\left[d_{l-1}^{-} d_{l}^{-} \tilde{\beta}\right]=0 .
$$

Clearly (5.21) and (5.22) follow from (5.23) and (5.24). This completes the proof of Lemma 5.5.

The short exact sequence (5.20) in Lemma 5.5 generates the following associated long exact sequence of the cohomology groups

$$
E_{l+p, 2}^{p-1, n} \rightarrow T_{l}^{n-p} \stackrel{\delta}{\rightarrow} C_{l}^{n-p} \rightarrow E_{l+p, 2}^{p, n} \rightarrow T_{l-1}^{n-(p+1)} \stackrel{\delta}{\rightarrow} C_{l-1}^{n-(p+1)} \rightarrow,
$$

where $\delta$ is the connecting homomorphism.

LEMMA 5.6. We have $\delta(x)=x$ for all $x \in T_{l-1}^{n-(p+1)}$ and for all $0 \leq p \leq n-1$.

Proof of Lemma 5.6. Let $x \in T_{l-1}^{n-(p+1)}$. Using (5.20) we write $x=\left[d_{l}^{-}\right] y, y \in$ $\left(\mathcal{P}^{n-p}\left(M^{2 n}\right)\right) / d_{l}^{+}\left(\mathcal{P}^{n-p-1}\left(M^{2 n}\right)\right)$. By definition of the connecting homomorphism we have $\delta x=\left[\hat{d}_{l}^{-} y\right]=x$. This completes the proof of Lemma 5.6.

Now let us complete the proof of Theorem 5.2. From Lemma 5.6 and the long exact sequence (5.25) we obtain $E_{l+p, 2}^{p, n}=C_{l}^{n-p} / T_{l}^{n-p}$. Taking into account (5.16) which defines $T_{l}^{n-p}$ to be the kernel of the surjective homomorphism $\left[L^{p}\right]: C_{l}^{n-p} \rightarrow$ $H_{l+p}^{n+p}\left(M^{2 n}\right)$, we conclude that

$$
E_{l+p, 2}^{p, n}=H_{l+p}^{n+p}\left(M^{2 n}\right) \text { for } 0 \leq p \leq n-1 .
$$

Next, by Lemma $4.1 E_{l, 1}^{n, n}=C^{\infty}\left(M^{2 n}\right)$. Since $d_{l, 1}\left(E_{l, 1}^{n, n}\right)=0$, using (5.19) we get

$$
E_{l+n, 2}^{n, n}=\frac{C^{\infty}\left(M^{2 n}\right)}{d_{l}^{-}\left(\mathcal{P}^{1}\left(M^{2 n}\right)\right)}=H^{0}\left(\mathcal{P}^{*}\left(M^{2 n}\right), d_{l}^{-}\right)
$$

By Proposition $3.11, d_{l}^{-}$is proportional to $\left(d_{l}\right)_{\omega}^{*}$. Applying the symplectic star operator we get from $(5.27)$

$$
E_{l+n, 2}^{n, n}=H^{0}\left(\mathcal{P}^{*}\left(M^{2 n}\right), d_{l}^{-}\right)=H^{0}\left(\mathcal{P}^{*}\left(M^{2 n}\right),\left(d_{l}\right)_{\omega}^{*}\right)=H_{n+l}^{2 n}\left(M^{2 n}\right) .
$$

Clearly the third assertion of Theorem 5.2 follows from (5.26) and (5.28). The last assertion of Theorem 5.2 follows immediately. This completes the proof of Theorem 5.2 .

From the exact sequence (5.5) we obtain immediately the following

Corollary 5.7. Assume that $\omega=d_{1} \tau$. For $0 \leq p \leq q \leq n-1$ we have

$$
E_{l+p, 1}^{p, q}=H_{l}^{q-p}\left(M^{2 n}\right) \oplus H_{l-1}^{q-p-1}\left(M^{2 n}\right) .
$$


Theorem 5.2 can be generalized as follows. Assume that $\omega^{p}=d_{T} \rho$ for some $\rho \in \Omega^{2 p-1}\left(M^{2 n}\right)$, in particular $d_{T} \omega^{p}=0$. Clearly $d_{p}\left(\omega^{p}\right)=0=d_{T}\left(\omega^{p}\right)$ implies that $T=p$, since $L^{p}: \Omega^{1}\left(M^{2 n}\right) \rightarrow \Omega^{1+2 p}\left(M^{2 n}\right)$ is injective. Furthermore we have $\omega^{k+T}=d_{k+T}(\rho \wedge \omega)$ for all $t \geq 0$. Thus there exists a minimal number $T$ such that $\omega^{T}=d_{T} \rho$ for some $\rho \in \Omega^{2 T-1}\left(M^{2 n}\right)$.

Theorem 5.8. (cf. [7, Theorem 3]) Assume that $\omega^{T}=d_{T} \rho$ and $T \geq 2$. Then the spectral sequence $\left(E_{l, r}^{p, q}, d_{l, r}\right)$ stabilizes at the term $E_{l, T+1}^{*, *}$.

Proof. Our proof of Theorem 5.8 exploits the construction of the exact couple associated with a filtered complex. We use many ideas from [8]. The main idea is to find a short exact sequence, whose middle term is $E_{l, T}^{*, *}$, and moreover, this short exact sequence is induced from the trivial action of the operator $L^{T}$ on (a part of) complexes entering in the derived exact couples (cf. with the proof of Theorem 5.2). The condition $T \geq 2$ is necessary for Lemma 5.9 below.

Let us begin with recalling the construction of the derived exact couple associated with a filtered complex $\left(F^{p} K^{*}, d_{l}\right)$ following [23, p.37-43]. We associate with a filtration $\left(F^{p} K^{*}, d_{l}\right)$ the following exact couple

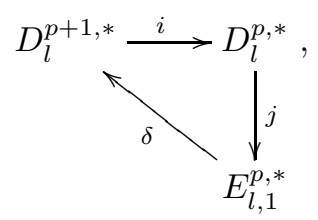

where $D_{l}^{p, q}:=H^{p+q}\left(F^{p} K^{*}, d_{l}\right)$, which we also abbreviate as $H_{l}^{p+q}\left(F^{p} K^{*}\right)$, and

$$
\rightarrow D_{l}^{p+1, q-1} \stackrel{i}{\rightarrow} D_{l}^{p, q} \stackrel{j}{\rightarrow} E_{l, 1}^{p, q} \stackrel{\delta}{\rightarrow} D_{l}^{p+1, q} \stackrel{i}{\rightarrow} D_{l, 1}^{p, q+1} \stackrel{j}{\rightarrow} E_{l, 1}^{p, q+1} \rightarrow
$$

is the long exact sequence of cohomology groups associated with the following short exact sequence of chain complexes

$$
0 \rightarrow\left(F^{p+1} K^{p+q}, d_{l}\right) \stackrel{\tilde{i}}{\rightarrow}\left(F^{p} K^{p+q}, d_{l}\right) \stackrel{\tilde{j}}{\rightarrow}\left(E_{l, 0}^{p, q}, d_{l, 0}\right) \rightarrow 0 .
$$

The differential $d_{l, 1}: E_{l, 1}^{p, q} \rightarrow E_{l, 1}^{p+1, q}$, defined in (5.1), satisfies the following relation: $d_{l, 1}=j \circ \delta$. We refer the reader to [23] for a comprehensive exposition on the relation between the spectral sequence of a filtration and its associated exact couple.

Set $\left(D_{l}^{*, *}\right)^{0}:=D_{l}^{*, *}$. We define the $t$-th derived exact couple of the exact couple (5.30), $t \geq 1$,

$$
\left(D_{l}^{p+1, q-1}\right)^{(t)} \stackrel{i^{(t)}}{\rightarrow}\left(D_{l}^{p-t, q+t}\right)^{(t)} \stackrel{j^{(t)}}{\rightarrow} E_{l, t+1}^{p, q} \stackrel{\delta^{(t)}}{\rightarrow}\left(D_{l}^{p+1, q}\right)^{(t)}
$$

inductively as follows [23, p. 38].

$$
\begin{array}{r}
\left(D_{l}^{p, q}\right)^{(t)}:=i\left(D_{l}^{p+1, q-1}\right)^{(t-1)} \subset D_{l}^{p, q}, \\
i^{(t)}\left(i^{t} x\right):=i\left(i^{t} x\right) \text { for } i^{t} x \in\left(D_{l}^{p, q}\right)^{(t)}, \\
E_{l, t+1}^{p, q}:=\frac{\operatorname{ker} d_{l, t} \cap E_{l, t}^{p, q}}{d_{l, t-1}\left(E_{l, t}^{p-t+1, q+t-2}\right)}, \\
j^{(t)}\left(i^{t} x\right):=\left[j^{(t-1)} \circ\left(i^{(t-1)} x\right)\right], \\
\delta^{(t)}([e]):=\delta^{(t-1)}(e) \in i\left(D_{l}^{p, q}\right)^{(t-1)}, \\
d_{l, t+1}:=j^{(t)} \circ \delta^{(t)} .
\end{array}
$$


Next we consider the following commutative diagram

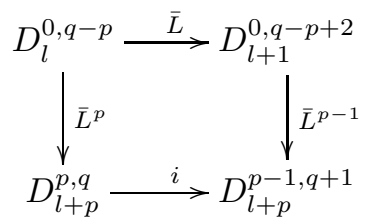

where $\bar{L}^{p}$ is induced by the linear operator $L^{p}: \Omega^{q-p}\left(M^{2 n}\right) \rightarrow \Omega^{q+p}\left(M^{2 n}\right)$.

The diagram (5.39) leads us to consider the following diagram

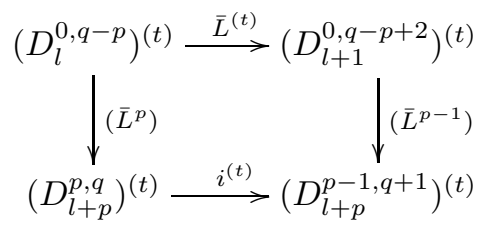

where $\bar{L}^{(t)}$ (resp. $\left.\left(\bar{L}^{p}\right)\right)$ is the restriction of $\bar{L}\left(\operatorname{resp} . \bar{L}^{p}\right)$ to $\left(D_{l+p}^{p, q}\right)^{(t)}$.

Lemma 5.9. For $t \geq 1$ and $p \geq 1, T \geq 2$, the following statements hold.

1. The diagram (5.40) is commutative.

2. $\left(\bar{L}^{p}\right)$ is an isomorphism.

3. $\operatorname{Im}\left(i^{(t)}\right)=\operatorname{Im}\left(\left(\bar{L}^{p-1}\right)\right)$.

4. $\left(\bar{L}^{(T-1)}\right)\left(D_{l}^{0, q-p}\right)^{(T-1)}=0$, if $d_{T} \omega^{T}=0$.

Proof. 1. The commutativity of (5.40) is an immediate consequence of the commutativity of the diagram (5.39).

2. We prove the second assertion of Lemma 5.9 by induction, beginning with $t=1$. Let $x=i(\alpha) \in\left(D_{l+p}^{p, q}\right)^{(1)}=i\left(D_{l+p}^{p+1, q-1}\right)=i\left(H_{l+p}^{p+q}\left(F^{p+1} K^{*}\right)\right) \subset D_{l+p}^{p, q}$. Then there is an element $\alpha^{\prime} \in \Omega^{q-p-2}\left(M^{2 n}\right)$ such that $\left[L^{p+1} \alpha^{\prime}\right]=\alpha \in D_{l+p}^{p+1, q-1}$, or equivalently $d_{l+p}\left(L^{p+1} \alpha^{\prime}\right)=0$. Hence $L^{p+1}\left(d_{l-1}\left(\alpha^{\prime}\right)\right)=0$. Since $d_{l-1} \alpha^{\prime} \in \Omega^{q-p-1}\left(M^{2 n}\right)$, $L^{p+1}\left(d_{l-1}\left(\alpha^{\prime}\right)\right)=0$ implies that $d_{l-1} \alpha^{\prime}=0$, so $\alpha^{\prime} \in H_{l-1}^{q-p-2}\left(M^{2 n}\right)$. Hence $x=\bar{L}^{p}\left(L \alpha^{\prime}\right)$, and $L \alpha^{\prime}=i\left(\alpha^{\prime}\right) \in\left(D_{l}^{0, q-p}\right)^{(1)}$. This proves that the linear map $\left(\bar{L}^{p}\right)$ is surjective for $t=1$. Furthermore, the map $\left(\bar{L}^{p}\right)$ is injective for $t=1$, since $L^{p}: \Omega^{q-p} M^{2 n} \rightarrow \Omega^{q+p} M^{2 n}$ is injective, and $L^{p} d_{l}=d_{l+p} L^{p}$. This proves Lemma 5.9.2 for $t=1$.

Now assume that Lemma 5.9.2 has been proved for $t=k$. Since $\left(D_{l}^{0, q-p}\right)^{(k+1)}$ is a subset of $\left(D_{l}^{0, q-p}\right)^{(k)}$ the injectivity of $\left(\bar{L}^{p}\right)$ follows from the inductive statement. The surjectivity of $\left(\bar{L}^{p}\right)$ also follows from the commutativity of the diagram (5.40), which implies that $\left(\bar{L}^{p-1}\right)$ maps the image $i\left(\left(D_{l}^{0, q-p}\right)^{(k)}\right)$ onto the set $i^{(k)}\left(D_{l+p}^{p, q}\right)^{(k)}=$ $\left(D_{l+p}^{p-1, q+1}\right)^{(k+1)}$. This proves Lemma 5.9.2 for all $t \geq 2$.

3. Clearly Lemma 5.9.3 is a consequence of Lemma 5.9.2 and the commutativity of the diagram (5.40).

4. Let us compute $\bar{L}^{(T-1)} \beta$ for $\beta \in\left(D_{l}^{0, q-p-2}\right)^{(T-1)}$, where $T \geq 2$. By definition $\bar{L}^{(T-1)} \beta=\bar{L}\left(i^{(T-1)}[\tilde{\beta}]\right)$, where $\tilde{\beta}=L^{T-1} \hat{\beta} \in \Omega^{q-p-2}\left(M^{2 n}\right)$ for some $\hat{\beta} \in \Omega^{q-p-2 T}\left(M^{2 n}\right)$, and $[\tilde{\beta}] \in D_{l}^{0, q-p}$, in particular we have $d_{l} \tilde{\beta}=0$. Thus $L^{T-1} d_{l-T-1} \tilde{\beta}=0$. Since $L^{T-1}: \Omega^{q-p-2 T+2}\left(M^{2 n}\right) \rightarrow \Omega^{q-p}\left(M^{2 n}\right)$ is injective we get $d_{l-T+1} \hat{\beta}=0$. Now we have

$$
\bar{L}^{(T-1)} \beta=\bar{L}\left(i^{(T-1)} \tilde{\beta}\right)=i^{(T-1)}\left(\left[L^{T} \hat{\beta}\right]\right) \in\left(D^{p-1, q+1}\right)_{l+p}^{(T)},
$$


by Lemma 5.9.1.

Note that

$$
\left[L^{T} \hat{\beta}\right]=\left[d_{T} \rho \wedge \hat{\beta}\right]=\left[d_{T+(l-T+1)}(\rho \wedge \hat{\beta})\right]=0 \in D_{l+1}^{0, q-p+2} .
$$

Clearly (5.41) and (5.42) imply the last assertion of Lemma 5.9.

Lemma 5.9 .4 and the $(T-1)$ th-derived exact couple yield the following short exact sequence

$$
0 \rightarrow\left(D_{l}^{p-(T-1), q+(T-1)}\right)^{(T-1)} \stackrel{j^{(T-1)}}{\rightarrow} E_{l, T}^{p, q} \stackrel{\delta^{(T-1)}}{\rightarrow}\left(D_{l}^{p+1, q}\right)^{(T-1)} \rightarrow 0 .
$$

LEMma 5.10. The short exact sequence (5.43) generates a short exact sequence of the following chain complexes

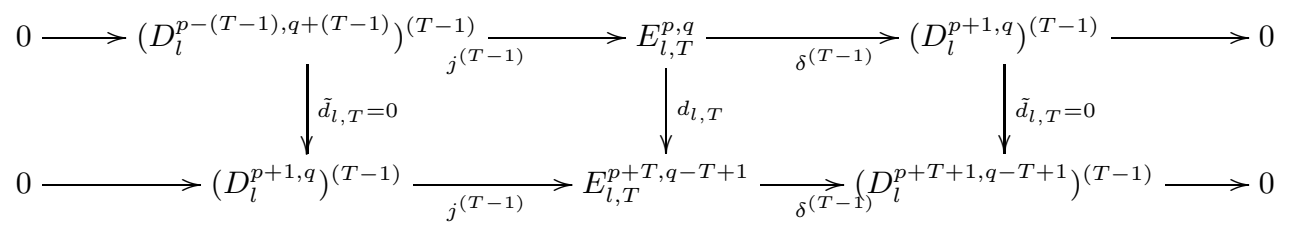

Proof. It suffices to show that

$$
\begin{gathered}
d_{l, T} j^{(T-1)}=0, \\
\delta^{(T-1)} d_{l, T}=0 .
\end{gathered}
$$

The equality $d_{l, T} j^{(T-1)}=0$ holds, since $d_{l, T}$ is a quotient map of the linear operator $d_{l}$ acting on $\Omega^{*}\left(M^{2 n}\right)$, and $j^{(T-1)}$ associates a cycle in $D_{l}^{p-(T-1), q+(T-1)} \subset$ $H_{l}^{p+q}\left(M^{2 n}\right)$ to its class in $E_{l, T}^{p, q}$.

The equality $\delta^{(T-1)} d_{l, T}=0$ holds, since $\delta^{(T-1)} d_{l, T}=\delta^{(T-1)} j^{(T-1)} \delta^{(T-1)}=0$. This completes the proof of Lemma 5.10.

Let us continue the proof of Theorem 5.8. From Lemma 5.10 we obtain the following long exact sequence of the associated cohomology groups

$$
\left(D_{l}^{p-(T-1), q+(T-1)}\right)^{(T-1)} \stackrel{j^{*}}{\rightarrow} E_{l, T+1}^{p, q} \stackrel{\delta^{*}}{\rightarrow}\left(D_{l}^{p+1, q}\right)^{(T-1)} \stackrel{\partial}{\rightarrow}\left(D_{l}^{p+1, q}\right)^{(T-1)} .
$$

Lemma 5.11. For $0 \leq p \leq q \leq n$ the connecting homomorphism $\partial$ : $\left(D_{l}^{p+1, q}\right)^{(T-1)} \rightarrow\left(D_{l}^{p+1, q}\right)^{(T-1)}$ in $(5.46)$ is equal to the identity.

Proof. By (5.43) for any $x \in\left(D_{l}^{p+1, q}\right)^{(T-1)}$ there exists $e \in E_{l, T}^{p, q}$ such that $y=$ $\delta^{(T-1)}(e)$. Since $d_{l, T}^{p, q}(e) \in \operatorname{ker} \delta^{(T-1)}$ there exists an element $y \in\left(D_{l}^{p+1, q}\right)^{(T-1)}$ such that $j^{(T-1)}(y)=d_{l, T}^{p, q}(e)=j^{(T-1)} \delta^{(T-1)}(e)$. Since $j^{(T-1)}$ is injective, $y=\delta^{(T-1)}(e)$. By definition $\partial(x)=y=\delta^{(T-1)}(e)$. It follows that $\partial\left(\delta^{(T-1)} e\right)=\delta^{(T-1)} e$. This completes the proof of Lemma 5.11.

Corollary 5.12. For $p \geq T$ we have $E_{l, T+1}^{p, q}=0$.

Proof. For $p \geq T$ Lemma 5.11 yields the following exact sequence

$$
\left(D_{l}^{*, *}\right)^{(T-1)} \stackrel{I d}{\rightarrow}\left(D_{l}^{*, *}\right)^{(T-1)} \stackrel{j^{*}}{\rightarrow} E_{l, T+1}^{p, q} \stackrel{\delta^{*}}{\rightarrow}\left(D_{l}^{*, *}\right)^{(T-1)} \stackrel{I d}{\rightarrow}\left(D_{l}^{*, *}\right)^{(T-1)}
$$


which implies Corollary 5.12 immediately.

It follows from Corollary 5.12 that $d_{l, T+1}: E_{l, T+1}^{p, q} \rightarrow E_{l, T+1}^{p+T+1, q-T}=0$ for all $p \geq 0$. This completes the proof of Theorem 5.8.

We end this section with presenting a proof of the following stabilization theorem.

Theorem 5.13. (cf. [7, Theorem 4]) Assume that $\left(M^{2 n}, \omega, \theta\right)$ is a compact connected globally conformal symplectic manifold. Then the spectral sequence $\left(E_{l, k}^{p, q}, d_{l, k}\right)$ stabilizes at the $E_{l, 2}^{*, *}$-term.

Proof. By Theorem 4.6 it suffices to prove Theorem 5.13 for the case of a symplectic manifold $\left(M^{2 n}, \omega\right)$, i.e. $\theta=0$. The proof we present here uses many ideas in the proof of Theorem 2 in Di Pietro's Ph.D. Thesis [8] stated for connected compact symplectic manifolds.

By Lemma $4.1 E_{l, 1}^{p, q}=0=E_{l, k}^{p, q}$ if $q<p$ or $q>n$ for all $k \geq 1$. Thus it suffices to examine the terms $E_{l, k}^{p, p}, E_{l, k}^{p, p+r}$, for $0 \leq p \leq n-r, r \geq 1, k \geq 2$.

LemMA 5.14. Assume that $\left(M^{2 n}, \omega, \theta\right)$ is a compact l.c.s. manifold, and $0 \leq p \leq$ $n$.

1. If $\left(M^{2 n}, \omega, \theta\right)$ is a globally conformal symplectic manifold, then $E_{l, k}^{p, p}=\mathbb{R}$ for all $l$ and $k \geq 2$. Moreover $E_{l, k}^{p, p}$ is generated by the $p$-th power of the symplectic form $\omega$.

2. If $\left(M^{2 n}, \omega, \theta\right)$ is not conformal equivalent to a symplectic manifold, then $E_{l, k}^{p, p}=0$ for all $p \neq l$ and for all $k \geq 1$.

Proof of Lemma 5.14. By (4.16) if $0 \leq p \leq n-1$ then

$$
E_{l, 1}^{p, p}=H_{l-p}^{0}\left(M^{2 n}\right) .
$$

By (4.8) we obtain

$$
E_{l, 1}^{n, n}=C_{l-n}^{0}=C^{\infty}\left(M^{2 n}\right) .
$$

We get from (5.48) and (5.2)

$$
E_{l, 2}^{n, n}=C^{\infty}\left(M^{2 n}\right) / d_{l, 1}\left(E_{l, 1}^{n-1, n}\right)=C^{\infty}\left(M^{2 n}\right) / d_{l-n}^{-}\left(\mathcal{P}^{1}\left(M^{2 n}\right)\right)=H^{0}\left(\mathcal{P}^{*}\left(M^{2 n}\right), d_{l-n}^{-}\right) .
$$

By Corollary 3.15

$$
H_{0}\left(\mathcal{P}^{*}\left(M^{2 n}\right), d_{l}^{-}\right)=H_{0}\left(\mathcal{P}^{*}\left(M^{2 n}\right),\left(d_{l-n}\right)_{\omega}^{*}\right)=H^{2 n}\left(\Omega^{*}\left(M^{2 n}\right), d_{l}\right) .
$$

Note that $d_{l, k}\left(E_{l, k}^{n, n}\right)=0$ and $\operatorname{Im} d_{l, k} \cap E_{l, k}^{n, n}=0$ for all $k \geq 2$. Using (5.49) we get

$$
E_{l, k}^{n, n}=E_{l, 2}^{n, n}=H_{0}\left(\mathcal{P}^{*}\left(M^{2 n}\right), d_{l}^{-}\right) \text {for all } k \geq 2 .
$$

Combining (5.51), (5.50) with Corollary 3.15 we obtain the assertion of Lemma 5.14 for the cases $p=0$ or $p=n$.

Now let us consider $E_{k, r}^{p, p}$ with $0<p<n$. By (4.16) $E_{l, 1}^{p, p}=H_{l-p}^{0}\left(M^{2 n}\right)$.

Let us first assume that $M^{2 n}$ is globally conformal symplectic. Using Theorem 4.6 we drop $l$ in the lower index of $d_{l, r}$ and $E_{l, r}^{p, q}$. First we note that $E_{0}^{p, p}$ is generated by $\omega^{p}$. Since $E_{k}^{p, p}$ is a quotient of $E_{0}^{p, p}$ and $\left[\omega^{p}\right] \in E_{k}^{p, p}$ for all $k \geq 1$, taking into account $\left[\omega^{n}\right] \neq 0 \in E_{k}^{n, n}$, we complete the proof of Lemma 5.14.1.

Now let us assume that $[\theta] \neq 0 \in H^{1}\left(M^{2 n}\right)$. Then Corollary 3.15 asserts that $H_{l}^{0}\left(M^{2 n}\right)=0$ for all $l \neq p$. It follows that $H_{l, \infty}^{p, p}=0$ for all $l \neq p$. This complete the proof of Lemma 5.14. 
LEMma 5.15. Assume that $\left(M^{2 n}, \omega\right)$ is a connected compact symplectic manifold. Then for $0 \leq p \leq n-2$ and $k \geq 2$ we have $E_{k}^{p, p+1}=H^{1}\left(M^{2 n}\right)$. Furthermore $E_{k}^{n-1, n}=E_{2}^{n-1, n}$ for all $k \geq 2$.

Proof of Lemma 5.15. Using (5.1) and (5.2) we note that $d_{1}: E_{1}^{0,1} \rightarrow E_{1}^{1,1}$ is equivalent to the map $d^{-}: H^{1}\left(\mathcal{P}^{*}\left(M^{2 n}\right), d^{+}\right) \rightarrow H^{0}\left(M^{2 n}, \mathbb{R}\right)=\mathbb{R}$. By $(3.21)$ $H^{1}\left(\mathcal{P}^{*}\left(M^{2 n}\right), d^{+}\right)=H^{1}\left(M^{2 n}\right)$. Hence

$$
E_{2}^{0,1}=H^{1}\left(M^{2 n}\right)
$$

It follows that, the image $d_{k}\left(E_{k}^{0,1}\right)=0$ for all $k \geq 2$. Thus we get from (5.52)

$$
E_{k}^{0,1}=H^{1}\left(M^{2 n}\right) \text { for all } k \geq 2 .
$$

This proves Lemma 5.15 for $E_{k}^{0,1}, k \geq 2$. Since the operator $L^{p}: \Omega^{1}\left(M^{2 n}\right) \rightarrow$ $\Omega^{2 p+1}\left(M^{2 n}\right)$ is injective for all $p \leq n-1$, using Lemma 5.14 we get

$$
E_{k}^{0,1} \cong E_{k}^{0,1} \wedge E_{k}^{p, p} \subset E_{k}^{p, p+1} \text { for all } k \geq 2
$$

Note that $E_{k}^{p, p+1}$ is a quotient of $E_{2}^{p, p+1}$, which is isomorphic to $E_{2}^{0,1}$ by Proposition 5.1. Taking into account (5.53) we obtain from (5.54)

$$
E_{k}^{p, p+1} \cong E_{2}^{0,1}=H^{1}\left(M^{2 n}\right) \text { for all } p \leq n-2 \text { and } k \geq 2 .
$$

This completes the proof the first assertion of Lemma 5.15. The second assertion of Lemma 5.15 follows from the observation that $d_{2}\left(E_{2}^{n-1, n}\right)=0=\operatorname{Im} d_{2} \cap E_{l, 2}^{n-1, n}$, and $d_{k}\left(E_{k}^{n-1, n}\right)=0=\operatorname{Im} d_{k} \cap E_{k}^{n-1, n}$ for all $k \geq 3$.

LEMMA 5.16. Assume that $\left(M^{2 n}, \omega\right)$ is a connected compact symplectic manifold. Then $E_{k}^{n-2, n}=E_{2}^{n-2, n}$ for all $k \geq 2$. Furthermore, for $0 \leq p \leq n-3$ and $k \geq 2$ we have

$$
E_{k}^{p, p+2} \cong E_{2}^{0,2}
$$

Proof. First we note that for $k \geq 2$

$$
\begin{aligned}
d_{k}\left(E_{k}^{n-2, n}\right) & =0, \\
\operatorname{Im} d_{k} \cap E_{k}^{n-2, n} & =0 .
\end{aligned}
$$

Hence

$$
E_{k}^{n-2, n}=E_{2}^{n-2, n} \text { for all } k \geq 2 .
$$

This proves the first assertion of Lemma 5.16. Next we observe that $d_{2}\left(E_{2}^{0,2}\right)=0$ and $\operatorname{Im} d_{2} \cap E_{2}^{0,2}=0$. Hence we get

$$
E_{k}^{0,2}=E_{2}^{0,2} \text { for all } k \geq 2 .
$$

Now we assume that $0 \leq p \leq n-3$. Since $L^{p}: \Omega^{2}\left(M^{2 n}\right) \rightarrow \Omega^{2+2 p}\left(M^{2 n}\right)$ is injective, using Lemma 5.14 we get

$$
E_{k}^{0,2} \cong E_{k}^{0,2} \wedge E_{k}^{p, p} \subset E_{k}^{p, p+2}
$$


Since $E_{k}^{p, p+2}$ is a quotient group of $E_{2}^{p, p+2}$, which is isomorphic to $E_{2}^{0,2}$ by Proposition 5.1 , using (5.58) and (5.59) we get

$$
E_{k}^{p, p+2} \cong E_{2}^{0,2} \text { for all } 0 \leq p \leq n-3
$$

This completes the proof of Lemma 5.16.

LemMA 5.17. We have $E_{k}^{p, p+r}=E_{2}^{0, r}$ for all $k \geq 2, p+r \leq n-1$ and $r \geq 3$. Furthermore $E_{k}^{n-r, n}=E_{2}^{n-r, n}$ for all $k \geq 2$ and $r \geq 3$.

Proof. We prove Lemma 5.17 inductively on $r$ beginning with $r=3$. For each $r$ we will consider $E_{k}^{p, p+r}$ with $k$ and $p$ increasing inductively. First we note that

$$
d_{2}\left(E_{2}^{0,3}\right)=0 \in E_{2}^{2,2},
$$

since $E_{2}^{2,2}=E_{k}^{2,2}$ for all $k \geq 2$ by Lemma 5.14 . From (5.61) we obtain easily

$$
E_{k}^{0,3}=E_{2}^{0,3} \text { for all } k \geq 2 .
$$

Now using the injectivity of the map $L^{p}: \Omega^{3}\left(M^{2 n}\right) \rightarrow \Omega^{2 p+3}\left(M^{2 n}\right)$ for $p \leq n-4$ and Lemma 5.14, we get from (5.62)

$$
E_{2}^{0,3}=E_{k}^{0,3}=E_{k}^{0,3} \wedge E_{k}^{p, p} \subset E_{k}^{p, p+3} .
$$

Since $E_{k}^{p, p+3}$ is a quotient group of $E_{2}^{p, p+3}=E_{2}^{0,3},(5.63)$ implies

$$
E_{k}^{p, p+3}=E_{2}^{0,3} \text { for all } 0 \leq p \leq n-4, k \geq 2 .
$$

This proves the first assertion of Lemma 5.17 for $r=3$. The second assertion of Lemma 5.17 for $r=3$ follows from the identities $\operatorname{Im} d_{k} \cap E_{k}^{n-3, n}=0$ and $d_{k}\left(E_{k}^{n-3, n}\right)=$ $0 \in E_{k}^{n+k-3, n-k+1}$ if $k \geq 2$, which is a consequence of Lemma 5.14 if $k=2$.

Repeating this procedure we have for each $n \geq r \geq 3$ the following sequences of identities with $k \geq 2$ and $0 \leq p \leq n-r$. First by induction on $r$ we get

$$
d_{2}\left(E_{2}^{0, r}\right)=0 \in E_{2}^{2, r-1},
$$

since $E_{2}^{2, r-1}=E_{k}^{2, r-1}$ for all $k \geq 2$ by the induction step. From (5.65) we obtain immediately

$$
E_{k}^{0, r}=E_{2}^{0, r} \text { for all } k \geq 2 .
$$

Since the map $L^{p}: \Omega^{r}\left(M^{2 n}\right) \rightarrow \Omega^{2 p+r}\left(M^{2 n}\right)$ for $p \leq n-r$ is injective, using Lemma 5.14 , we get from $(5.66)$

$$
E_{2}^{0, r}=E_{k}^{0, r}=E_{k}^{0, r} \wedge E_{k}^{p, p} \subset E_{k}^{p, p+r} .
$$

Since $E_{k}^{p, p+r}$ is a quotient group of $E_{2}^{p, p+r}$ which is isomorphic to $E_{2}^{0, r}$ if $p+r \leq n-1$ by Proposition 5.1, (5.67) implies

$$
E_{k}^{p, p+r}=E_{2}^{0, r} \text { for all } 0 \leq p \leq n-r-1, k \geq 2 .
$$

Thus we get

$$
E_{k}^{0, r}=E_{k}^{p, p+r} \text { for all } 0 \leq p \leq n-r-1, k \geq 2
$$


This completes the proof of the first assertion of Lemma 5.17.

The second assertion of Lemma 5.17 for the inductive $r$ follows from the identities $\operatorname{Im} d_{k} \cap E_{k}^{n-r, n}=0$ and $d_{k}\left(E_{k}^{n-3, n}\right)=0 \in E_{k}^{n+k-r, n-k+1}$ if $k \geq 2$, which is a consequence of the induction assumption that $E_{k}^{n+k-r, n-k+1}=E_{2}^{n+k-r, n-k+1}$ for $0 \leq k \leq r-1$.

Clearly Theorem 5.13 follows from Lemmata 5.14, 5.15, 5.16 , 5.17.

REMARK 5.18. 1. Our stabilization theorem 5.13 gives an answer to the TsengYau question on the relation between the group $H^{p}\left(M^{2 n}, d^{+}\right)=E_{1}^{0, p}\left(M^{2 n}\right)$ for $0 \leq$ $p \leq n-1$ and the cohomology groups $H^{*}\left(M^{2 n}, \mathbb{R}\right)$.

2. In the next section we show that if $\left(M^{2 n}, \omega\right)$ is a compact Kähler manifold, then the spectral sequence stabilizes already at $E_{1}$-terms, see Theorem 6.2.

6. Kähler manifolds. In this section we prove that the spectral sequence $E_{r}^{p, q}$ stabilizes at the term $E_{1}^{*, *}$, if $\left(M^{2 n}, J, g\right)$ is a compact Kähler manifold and $\omega$ is the associated symplectic form (Theorem 6.2).

Let $\left(M^{2 n}, J, g, \omega\right)$ be a compact Kähler manifold. As before, denote by $d^{*}$ the formal adjoint of $d$. Since the operator $L$ commutes with the Laplacian $\triangle_{d}:=d d^{*}+$ $d^{*} d$ we get the induced Lefschetz decomposition of the space of harmonic forms on $M^{2 n}$, and hence the induced Lefschetz decomposition of $H^{*}\left(M^{2 n}, \mathbb{R}\right)$. Let us denote by $P H^{q}\left(M^{2 n}, \mathbb{R}\right)$ the subset of primitive cohomology classes in $H^{q}\left(M^{2 n}, \mathbb{R}\right)$. Note that each primitive cohomology class $[\alpha] \in P H^{q}\left(M^{2 n}\right)$ has a representative which is $\triangle_{d}$-harmonic and primitive.

Proposition 6.1. ([29, Proposition 3.18]) Assume that $\left(M^{2 n}, J, g, \omega\right)$ is a compact Kähler manifold. For $q \leq n-1$ we have $H_{q}\left(\mathcal{P}^{*}\left(M^{2 n}\right),(d)_{\omega}^{*}\right)=P H^{q}\left(M^{2 n}\right)=$ $H^{q}\left(\mathcal{P}^{*}\left(M^{2 n}\right), d^{+}\right)$.

Proof. We give here another proof using [4]. Bouche proved that if $\left(M^{2 n}, J, g, \omega\right)$ is a compact Kähler manifold, the coeffective cohomology groups $H^{2 n-q}\left(\mathcal{C}^{*} M^{2 n}, d\right)$ is isomorphic to the subgroup $H_{\omega}^{2 n-q}\left(M^{2 n}\right):=\left\{x \in H^{2 n-q}\left(M^{2 n}, \mathbb{R}\right) \mid L x=0\right\}$ for $0 \leq q \leq n-1$ [4, Proposition 3.1]. Next, using [5, Corollary 2.4.2], or the following formula: $\mathcal{J} d^{*} \mathcal{J}^{-1}=d_{\omega}^{*}$, which is proved in the similar way as (3.39) replacing (3.38) in the proof with (3.36), we observe that the symplectic star operator $*_{\omega}$ maps $H_{\omega}^{2 n-q}\left(M^{2 n}\right)$ isomorphically onto the group $P H^{q}\left(M^{2 n}\right)$. As we have noted in Remark 3.16.1, the coeffective cohomology group $H^{q}\left(\mathcal{C}^{*} M^{2 n}, d\right)$ is isomorphic to the primitive homology group $H_{q}\left(\mathcal{P}^{*} M^{2 n}\right)$. On the other hand Tseng-Yau proved that the group $H\left(\mathcal{P}^{*} M^{2 n},(d)_{\omega}^{-}\right)$is isomorphic to the group $H_{q}\left(\mathcal{P}^{*}\left(M^{2 n}\right), d^{-}\right)$[29, Lemma 2.7, part II] as well as to the group $H^{q,+}\left(M^{2 n}\right)$, [29, Proposition 3.5, part II], see also Proposition 3.11 and Proposition 3.14 above. Combining these observations we complete the proof of Proposition 6.1.

Theorem 6.2. Assume that $\left(M^{2 n}, J, g, \omega\right)$ is a compact Kähler manifold. Then the spectral sequence $E_{r}^{p, q}$ stabilizes at $E_{1}^{*, *}$.

Proof. Since $\left(M^{2 n}, J, g, \omega\right)$ is a compact symplectic manifold, by Theorem 5.13 the spectral sequence $E_{r}^{p, q}$ stabilizes at $E_{2}$-terms. Thus to prove Theorem 6.2 it suffices to show that all the differentials $d_{1}: E_{1}^{p, q} \rightarrow E_{1}^{p+1, q}$ vanish. By (5.2), if $q \leq n-1$ then $d_{1}: E_{1}^{p, q} \rightarrow E_{1}^{p+1, q}$ is defined by the image of $d^{-} \tilde{\alpha}, \tilde{\alpha} \in \mathcal{P}^{q-p}\left(M^{2 n}\right)$. In this case it suffices to show that any element $[\alpha] \in H^{q-p}\left(\mathcal{P}^{*}\left(M^{2 n}\right), d^{+}\right)$has a representative $\alpha \in \mathcal{P}^{q-p}\left(M^{2 n}\right)$ such that $d^{-} \alpha=0$. By the Hodge theory for $d^{+}$there is a harmonic representative $\alpha$ of $[\alpha]$ such that $\left(d^{+}\right)^{*} \alpha=0$. Lemma 3.13 implies that 
for such harmonic form $\alpha$ we have $d^{-} \alpha=0$. This implies $d_{1}\left(E_{1}^{p, q}\right)=0$ for $q \leq n-1$. It remains to consider the image $d_{1}\left(E_{1}^{p, n}\right)$. By (5.19) it suffices to show that any element $[\alpha] \in E_{1}^{p, n}$ has a representative $\alpha \in \mathcal{P}^{n-p}\left(M^{2 n}\right)$ such that $d^{-}(\alpha)=0$. Using the Hodge theory for $d^{+}$and (5.19) we choose $\alpha$ to be the harmonic form. By Lemma $3.13 d^{-}(\alpha)=0$. This completes the proof of Theorem 6.2 .

7. Examples. In this section we consider two simple examples of compact l.c.s. manifolds and their primitive cohomologies. The first example is a nilmanifold of Heisenberg type [27], the second example is a 4-dimensional solvmanifold described in $[1],[2],[26],[15]$. We calculate the primitive cohomology of these examples (Propositions $7.1,7.2$ ). We study some properties of primitive cohomology groups of a l.c.s. manifold, which is a mapping torus of a co-orientation preserving contactomorphism (Proposition 7.4). We show that the 4-dimensional solvmanifold is a mapping torus of a coorientation preserving contactomorphism of a connected contact 3-manifold, which is not isotopic to the identity (Theorem 7.6).

Let $H(n)$ denote the $(2 n+1)$-dimensional Heisenberg Lie group and $\Gamma$ its lattice. It is well-known that the nilmanifold $N^{2 n+2}:=(H(n) / \Gamma) \times S^{1}$ has a canonical l.c.s. form $\Omega$, which we now describe following [27]. Note that the Lie algebra $\mathfrak{h}(n) \oplus \mathbb{R}$ of $H(n) \times \mathbb{R}$ is given by $\left\langle X_{i}, Y_{i}, Z, A:\left[X_{i}, Y_{i}\right]=Z\right\rangle_{\mathbb{R}}$. We denote by $x_{i}, y_{i}, z, \alpha$ the dual basis. Clearly $d \alpha=0$ and $d \Omega=\alpha \wedge \Omega$. Here we use the same notations for the extension of $X_{i}, Y_{i}, Z, A, x_{i}, y_{i}, z, \alpha, \Omega$ to the right-invariants vector fields or differential forms on $H(n) \times \mathbb{R}$, as well as for the descending vector fields or differential forms on $N^{2 n+2}$.

Proposition 7.1. Let $\left(N^{2 n+2}, \Omega, \alpha\right)$ be the l.c.s. nilmanifold described above. All the Lichnerowicz-Novikov cohomology groups $H^{*}\left(\Omega^{*}\left(N^{2 n+2}\right), d_{k \alpha}\right)$ vanish, if $k \neq 0$. Consequently for $k \neq 0$ all the groups $E_{k, r}^{p, q}, r \geq 1$, of the associated spectral sequences vanish, unless $q=n$ and $r=1$. The group $E_{k, 1}^{p, n}$ is infinite dimensional for all $0 \leq p \leq n$.

Proof. The first assertion of Proposition 7.1 is a consequence of a result due to Millionshchikov, who proved that the Lichnerowicz-Novikov cohomology groups $H^{*}\left(\Omega^{*}(M), d_{\theta}\right)$ of a compact nilmanifold $M$ always vanish unless $\theta$ presents a trivial cohomology class in $H^{1}(M, \mathbb{R})$ [24, Corollary 4.2]. The second assertion of Proposition 7.1 for $E_{k, 1}^{p, q}$ is a consequence of the first assertion, combining with Lemma 4.1 and the exact sequence (4.12). Since $\Omega=d_{\alpha}(z)$, applying Theorem 5.2 we obtain the second assertion from the first assertion combining with the particular case $r=1$ proved above. The third assertion follows from Lemma 4.1 and from the ellipticity of the operators $d_{k}^{+}$, see the proof of Proposition 3.14. This completes the proof of Proposition 7.1.

Now we shall show an example of a l.c.s. 4-manifold $M_{k, n}$, which is an Inoue surface of type $S^{-}$, whose primitive cohomologies are non-trivial, and we will explain an implication of this non-triviality. The 4-manifold $M_{n, k}$ has been described in [1], $[2],[26],[15]$. Here we follow the exposition in [2]. Let $G_{k}$ be the group of matrices of the form

$$
\left(\begin{array}{llll}
e^{k z} & 0 & 0 & x \\
0 & e^{-k z} & 0 & y \\
0 & 0 & 1 & z \\
0 & 0 & 0 & 1
\end{array}\right)
$$


where $x, y, z \in \mathbb{R}$ and $k \in \mathbb{R}$ such that $e^{k}+e^{-k} \in \mathbb{Z} \backslash\{2\}$. The group $G_{k}$ is a connected solvable Lie group with a basis of right invariant 1-forms

$$
d x-k x d z, d y+k y d z, d z .
$$

There exists a discrete subgroup $\Gamma_{k} \subset G_{k}$ such that $N_{k}=G_{k} / \Gamma_{k}$ is compact. The basis (7.1) descends to a basis of 1 -forms $\alpha, \beta, \gamma$ on $N_{k}$. The forms $\gamma$ and $\alpha \wedge \beta$ are closed and their cohomology classes generate $H^{1}(M, \mathbb{R})$ and $H^{2}(M, \mathbb{R})$ respectively.

Now let $\lambda \in \mathbb{R}$ be a number such that $\lambda[\alpha \wedge \beta] \in H^{2}(M, \mathbb{Z})$. For given $k, n$ denote by $M_{k, n}$ the total space of the $S^{1}$-principal bundle over $N_{k}$ with the Chern class $n \lambda[\alpha \wedge \beta]$. Let $\eta$ be a connection form on $M_{k, n}$, equivalently

$$
d \eta=n \lambda(\alpha \wedge \beta)
$$

For simplicity we will denote the pull back to $M_{k, n}$ by the projection $M_{k, n} \rightarrow N_{k}$ of a form $\theta$ on $N_{k}$ again by $\theta$. Banyaga showed that $M_{k, n}$ possesses many interesting l.c.s. structures. Here we consider only two l.c.s. forms $d_{-k \gamma} \eta=n \lambda(\alpha \wedge \beta)-k \gamma \wedge \eta$ and $d_{k \gamma} \eta=n \lambda(\alpha \wedge \beta)+k \gamma \wedge \eta$ discovered by Banyaga [2, Remark 2]. Note that $M_{k, n}$ carries no symplectic structure, since $H^{2}\left(M_{k, n}, \mathbb{R}\right)=0[1]$. Since $M_{k, n}$ is compact, the Hodge theory applied to $d_{ \pm \gamma}$ yields that $H^{2-i}\left(\Omega^{*}\left(M_{n, k}\right), d_{ \pm k \gamma}\right)=H^{2+i}\left(\Omega^{*}\left(M_{n, k}\right), d_{ \pm k \gamma}\right)$. Since $[ \pm k \gamma] \neq 0 \in H^{1}\left(M_{n, k}, \mathbb{R}\right)$, the Lichnerowicz deformed differential $d_{ \pm k \lambda}$ is not gauge equivalent to the canonical differential $d$. Hence $H^{0}\left(\Omega^{*}\left(M^{2 n}\right), d_{ \pm k \gamma}\right)=0$. Denote by $\mathcal{P}_{ \pm}^{*}\left(M^{2 n}\right)$ the space of primitive forms corresponding to the l.c.s. form $d_{ \pm k \gamma} \eta$. Corollary 3.15 yields that $H^{0}\left(\mathcal{P}_{ \pm}^{*}\left(M_{k, n}\right), d_{ \pm l k \gamma}^{+}\right)=0$ for all $l \neq 0$, and $H^{0}\left(\mathcal{P}_{ \pm}^{*}\left(M^{2 n}\right), d\right)=\mathbb{R}$.

Proposition 7.2. 1. $H^{1}\left(\Omega^{*}\left(M_{k, n}\right), d_{ \pm k \gamma}\right)=\mathbb{R}$.

2. $H^{2}\left(\Omega^{*}\left(M_{k, n}\right), d_{ \pm k \gamma}\right)=\mathbb{R}$.

3. $H^{1}\left(\mathcal{P}_{ \pm}^{*}\left(M_{k, n}\right), d_{ \pm k \gamma}^{+}\right)=\mathbb{R}^{2}$.

Proof. It is known that $M_{k, n}$ is a complete solvmanifold. Indeed, the algebra $\mathfrak{g}_{k, n}$ of the corresponding solvable group possesses the basis $(X, Y, Z, T)$ dual to $(\alpha, \beta, \gamma, \eta)$ with the following properties [1], or see (7.5) and (7.6) below.

$$
\begin{array}{r}
{[X, Z]=k X,[X, Y]=-n \lambda T,[Y, Z]=-k Y,} \\
{[X, T]=[Y, T]=[Z, T]=0 .}
\end{array}
$$

Using (7.3) and (7.4) we observe that the Lie subalgebras $\langle T\rangle_{\mathbb{R}} \subset\langle T, X\rangle_{\mathbb{R}} \subset$ $\langle T, X, Y\rangle_{\mathbb{R}}$ are ideals of $\mathfrak{g}_{k, n}$, so $M_{k, n}$ is completely solvable. Now we apply the result by Millionshchikov [24, Corollary 4.1, Theorem 4.5], which reduces the computation of the Novikov cohomology groups of a compact complete solvmanifold $G / \Gamma$ to the computation of the induced Novikov cohomology groups of the Lie algebra $\mathfrak{g}$ of $G$. For our computation it is useful to rewrite (7.3) and (7.4) in the dual basis of $\mathfrak{g}_{k, n}^{*}$, or using the explicit formulae for $\alpha, \beta, \gamma, \eta$ given in (7.1), (7.2) above to obtain

$$
\begin{array}{r}
d \alpha=-k \alpha \wedge \gamma, d \beta=k \beta \wedge \gamma \\
d \gamma=0, d \eta=n \lambda(\alpha \wedge \beta) .
\end{array}
$$

1. Abbreviate $d_{ \pm k \gamma}$ as $d_{ \pm k}$. Using (7.5) and (7.6) we get

$$
\begin{gathered}
d_{ \pm k} \alpha=(k \pm k) \gamma \wedge \alpha, d_{ \pm k} \beta=(-k \pm k) \gamma \wedge \beta \\
\gamma=d_{ \pm k}( \pm 1 / k), d_{ \pm} \eta=n \lambda(\alpha \wedge \beta) \pm k \gamma \wedge \eta
\end{gathered}
$$


Using (7.7) and (7.8) it is easy to compute that $\alpha$ is a generator of $H^{1}\left(\Omega^{*}\left(M_{k, n}\right), d_{-k}\right)$, and $\beta$ is a generator of $H^{1}\left(\Omega^{*}\left(M_{k, n}\right), d_{k}\right)$. This proves the first assertion of Proposition 7.2 .

2. For computing $H^{2}\left(\Omega^{*}\left(M_{k, n}\right), d_{ \pm k}\right)$ we use $(7.7)$, (7.8), and the following formulae

$$
\begin{array}{r}
d_{ \pm k}(\alpha \wedge \beta)= \pm k \alpha \wedge \beta \wedge \gamma \\
d_{-k}(\alpha \wedge \gamma)=0, d_{k}(\beta \wedge \gamma)=0 \\
d_{ \pm k}(\alpha \wedge \eta)=(-k \pm k) \gamma \wedge \alpha \wedge \eta, d_{ \pm k}(\beta \wedge \eta)=(k \pm k) \gamma \wedge \beta \wedge \eta \\
d_{ \pm k}(\gamma \wedge \eta)=-n \lambda \alpha \wedge \beta \wedge \gamma
\end{array}
$$

It is easy to see that $\alpha \wedge \eta$ is a generator of $H^{2}\left(\Omega^{*}\left(M_{k, n}\right), d_{-k}\right)$ and $\beta \wedge \eta$ is a generator of $H^{2}\left(\Omega^{*}\left(M_{k, n}\right), d_{k}\right)$. This proves the second assertions of Proposition 7.2.

3. The third assertion of Proposition 7.2 is a consequence of the first assertion and Formula(3.22). This completes the proof of Proposition 7.2.

In the remaining part of this section we study some properties of primitive cohomology groups of l.c.s. manifolds associated with a co-orientation preserving contactomorphism. We show that the l.c.s. solvmanifold studied before is an example of a l.c.s. manifold associated with a non-trivial contactomorphism.

Let $\left(M^{2 n+1}, \alpha\right)$ be a co-orientable contact manifold and $f$ be a co-orientation preserving contactomorphism of $\left(M^{2 n+1}, \alpha\right)$, i.e. $f^{*}(\alpha)=e^{h} \cdot \alpha$ for some $h \in C^{\infty}\left(M^{2 n}\right)$. The mapping torus $M_{f}^{2 n+2}=(M \times[0,1]) /([x, 0]=[f(x), 1])$ of a contactomorphism $f$ is a fibration over $S^{1}$ whose fiber is $M^{2 n+1}$. Let us denote this fibration by $\pi: M_{f}^{2 n+2} \rightarrow S^{1}$ with $\pi^{-1}(s)=[M, s]$. Let $f_{t}: M_{f}^{2 n+2} \rightarrow M_{f}^{2 n+2}$ be a 1-parameter family of diffeomorphisms defined by:

$$
f_{t}([x, s])=[x, s+t \quad \bmod 1] \text { for } t \in \mathbb{R} .
$$

In particular $\left.f_{1}([x, 0])=[f(x), 0]\right)$. Let us also denote by $\alpha$ the contact 1 -form on $[M, 0]$ obtained by identifying $M$ with $[M, 0]$. Let $B$ be the vector field on $M_{f}^{2 n+2}$ defined by $B([x, s])=(d / d t)_{\mid t=0} f_{t}([x, s])$. Since $f^{*}(\alpha)=e^{h} \alpha$ the following 1 -form $\tilde{\alpha}$

$$
\tilde{\alpha}(x, t)_{\mid \pi^{-1}(t)}:=e^{-t h(x)} f_{-t}^{*}(\alpha), \tilde{\alpha}(B)=0 .
$$

is well-defined on $M_{f}^{2 n+2}$, moreover

$$
f_{t}^{*}(\tilde{\alpha})_{\mid \pi^{-1}(t)}=\tilde{\alpha}_{\mid \pi^{-1}(0)} \text { for all } 0 \leq t \leq 1 .
$$

Set $\theta:=\pi^{*}(d t)$.

Proposition 7.3. (cf. [3, Proposition 3.3.]) 1. Assume that $\left(M^{2 n+1}, \alpha\right)$ is a compact co-orientable contact manifold and $f$ is a co-orientation preserving contactomorphism. There exists a positive number $c_{0}$ such that $\left(M_{f}^{2 n+2}, \omega_{c}:=d \tilde{\alpha}+c \theta \wedge \tilde{\alpha}, c \theta\right)$ is a l.c.s. manifold for all $c \geq c_{0}$.

2. Assume that $f$ preserves the contact 1-form $\alpha$. Then $\left(M_{f}^{2 n+2}, \omega:=d \tilde{\alpha}+\theta \wedge \tilde{\alpha}, \theta\right)$ is a l.c.s. manifold.

Proof. 1. Clearly (7.9) implies that $r k d \tilde{\alpha} \geq r k d \alpha=2 n$. Using this we conclude that there exists a positive number $c_{0}$ such that $r k d \omega_{c}=2 n+2$ for all $c \geq c_{0}$, since $M^{2 n+1}$ is compact. Further, $d(c \theta)=0$ and $\omega_{c}=d_{c \theta}(\tilde{\alpha})$. This proves that $\left(M_{f}^{2 n+2}, \omega_{c}, c \theta\right)$ is a l.c.s. manifold. 
2. Assume that $f^{*}(\alpha)=\alpha$. Then $\tilde{\alpha}([x, t])_{\pi^{-1}(t)}=f_{-t}^{*} \alpha$. It follows that $r k d \tilde{\alpha}=$ $r k d \alpha=2 n$, and $r k \omega_{1}=2 n+2$. Hence $\omega_{1}=\omega$ is a l.c.s. form, taking into account $\omega=d_{\theta} \alpha$.

Proposition 7.4. 1. Suppose that $f_{0}$ and $f_{1}$ are co-orientation preserving contactomorphisms of a compact co-orientable contact manifold $\left(M^{2 n+1}, \alpha\right)$. The l.c.s. manifolds $M_{f_{0}}^{2 n+2}$ and $M_{f_{1}}^{2 n+2}$ are diffeomorphic, if $f_{0}$ and $f_{1}$ are isotopic. For sufficiently large number $c$ the primitive cohomology groups of $\left(M_{f_{0}}^{2 n+2}, \omega_{c}, c \theta\right)$ and of $\left(M_{f_{1}}^{2 n+2}, \omega_{c}^{\prime}, c \theta\right)$ are isomorphic.

2. Let $\theta$ be the Lee form of the associated l.c.s form on $M_{f}^{2 n+1}$. If $f$ is isotopic to the identity, the Lichnerowicz cohomology groups $H^{*}\left(\Omega^{*}\left(M_{f}^{2 n+2}\right), d_{c \theta}\right)$ are zero, for any $c \neq 0$.

Proof. The first assertion of Proposition 7.4.1 is well-known. The second assertion of Proposition 7.4.1 is a consequence of Theorem 4.6, observing that $\omega_{c}-\omega_{c}^{\prime}=d_{c \theta}(\tilde{\alpha}-$ $\left.\tilde{\alpha}^{\prime}\right)$.

Finally Proposition 7.4.2 follows from the first assertion, combining with the fact that the l.c.s. manifold $\left(M^{2 n+1} \times S^{1}, d_{\theta} \tilde{\alpha}, \theta\right)$ associated to the identity mapping of the contact manifold $\left(M^{2 n+1}, \alpha\right)$ has vanishing Lichnerowicz-Novikov groups, taking into account the Künneth formula and the formula $H^{*}\left(\Omega^{*}\left(S^{1}\right), d_{c d t}\right)=0$ if $c \neq 0$. This completes the proof of Proposition 7.4.

Now we shall show that our l.c.s. manifold $\left(M_{k, n}, d_{k \gamma} \eta, k \gamma\right)$ is a mapping torus of a non-trivial co-orientation preserving contactomorphism. First we prove the following

Proposition 7.5. Assume that $\gamma$ is a closed 1-form on a compact smooth manifold $M$. If $[\gamma] \in H^{1}(M, \mathbb{Z})$ and $\gamma$ is now-where vanishing, then there is a submersion $f: M \rightarrow S^{1}$ such that $f^{*}(d t)=\gamma$, where $d t$ is the canonical 1-form on $S^{1}$.

Proof. We use Tischler's argument in [28]. Since $S^{1}$ is the Eilenberg-Maclane space there exists a map $f_{1}: M \rightarrow S^{1}$ such that $f^{*}([d t])=[\gamma]$. Without loss of generality we assume that $f$ is a smooth map. Hence we have $f^{*}(d t)=\gamma+d h$ for some smooth function $h$ on $M$. Now we observe that $f_{1}^{*}(d t)+d h=\left(f_{1}+\Pi \circ h\right)(d t)$, where $\Pi: \mathbb{R} \rightarrow S^{1}$ is the natural projection. Clearly the map $f=f_{1}+\Pi \circ h$ is a submersion, since $\gamma$ is no-where vanishing. This completes the proof of Proposition 7.5 .

Now we are ready to show the following implication of Proposition 7.2.

THEOREM 7.6. The l.c.s. manifold $\left(M_{k, n}, d_{k \gamma} \eta, k \gamma\right)$ is a mapping torus of a coorientation preserving contactomorphism $f$ of a 3-dimensional connected contact manifold. Moreover $f$ is not isotopic to the identity.

Proof. Since $H^{1}\left(M_{k, n}, \mathbb{R}\right)=\mathbb{R}[1]$, and $d \gamma=0$, there exists a positive number $p$ such that $p[\gamma]$ is a generator of $H^{1}\left(M_{k, n}, \mathbb{Z}\right)=\mathbb{Z}=\operatorname{Hom}\left(H_{1}\left(M_{k, n}, \mathbb{Z}\right), \mathbb{Z}\right)[9$, Chapter VI, 7.22]. Applying Proposition 7.5 we conclude that $M_{k, n}$ is a fibration over $S^{1}$ whose fibers are the foliation $\mathcal{F}_{1}:=\{\gamma=0\}$, and $f^{*}(d t)=p \cdot \gamma$, since $\gamma$ is nowhere vanishing. Denote by $\pi: M_{k, n} \rightarrow S^{1}$ the corresponding fibration. Note that the restriction of $\eta$ to each fiber $\pi^{-1}(t), t \in S^{1}$, is a contact form, since $X, Y, T$ are tangent to the fiber and we have $\eta(T)=1, d \eta(X, Y) \neq 0$.

First we will show that the fiber $F:=\pi^{-1}(t), t \in S^{1}$, is connected. Let us consider the following exact sequence of homotopy groups

$$
\pi_{1}\left(M_{k, n}\right) \rightarrow \pi_{1}\left(S^{1}\right) \rightarrow \pi_{0}(F) \rightarrow 0=\pi_{0}\left(M_{k, n}\right) .
$$


To show that $\pi_{0}(F)=0$ it suffices to prove that the map $\pi_{1}\left(M_{k, n}\right) \rightarrow \pi_{1}\left(S^{1}\right)$ is surjective. Since $p[\gamma]$ is a generator of $H^{1}\left(M_{k, n}, \mathbb{Z}\right)$ there exists an element $a \in$ $H_{1}\left(M_{k, n}, \mathbb{Z}\right)$ such that $\langle p[\gamma], a\rangle=1$. Since $\left\langle[d t], \pi_{*}(a)\right\rangle=\langle p[\gamma], a\rangle=1$, it follows that $\pi_{*}: H_{1}\left(M_{k, n}, \mathbb{Z}\right) \rightarrow H_{1}\left(S^{1}\right)$ is surjective. Hence $\pi_{*}: \pi_{1}\left(M_{k, n}\right) \rightarrow \pi_{1}\left(S^{1}\right)$ is surjective. Hence $F$ is connected.

Now let $f_{t}$ denote the flow on $M_{k, n}$ generated by the vector field $Z$. We note that $\mathcal{L}_{Z}(\gamma)=d(\gamma(Z))=0$, so $f_{t}$ respects fibration $\pi$. Next we have $\left.\mathcal{L}_{Z}(\eta)=Z\right\rfloor n \lambda \alpha \wedge \beta+$ $d(\eta(Z))=0$. Hence $f_{t}$ preserves also the contact form on the fiber $F$. This proves the first assertion.

The second assertion is a consequence of Proposition 7.2 and Proposition 7.4. This completes the proof of Theorem 7.6.

Acknowledgement. H.V.L. thanks Alexandre Vinogradov for explaining the idea of their paper [7] during the conference on integrable systems at Hradec nad Moravici in October 2010. We acknowledge Alexandre Vinogradov kindness for lending H.V.L. the Ph.D. Thesis of Di Pietro in early summer 2011, which accelerated our work over sections 4 and 5 considerably. H.V.L. also thanks Thiery Bouche for sending his reprint [4], Petr Somberg for informing her of the paper by Rumin [25], Jürgen Jost for his support and his invitation to give a lecture on this subject at the Max-Planck-Institute in Leipzig in March 2011, and the ASSMS, Government College University, Lahore-Pakistan for their hospitality and financial support during her visits in February and September 2011, where a part of this note has been written. Last but not least, H.V.L. would like to express her gratitude to Kaoru Ono and Lorenz Schwachhofer for their helpful remarks.

\section{REFERENCES}

[1] L. C. De Andres, L. A. Cordero, M. Fernandez, And J. Mencia, Examples of fourdimensional compact locally conformal Kähler solvmanifolds, Geometriae Dedicata, 29 (1989), pp. 227-232.

[2] A. BAnYagA, Examples of non $d_{\omega}$-exact locally conformal symplectic forms, J. Geom., 87 (2007), pp. 1-13.

[3] G. Bande And D. Kotschick, Contact pairs and locally conformal symplectic structures, Harmonic maps and differential geometry, pp. 85-98, Contemp. Math., 542, Amer. Math. Soc., Providence, RI, 2011, arXiv.1006.0315.

[4] T. Bouche, La cohomologie effective d'une variété symplectique, Bull. Sci. Math. 2.serie, 114 (1990), pp. 115-122.

[5] J. C. BRylinski, A differential complex for Poisson manifolds, JDG, 28 (1988), pp. 93-114.

[6] D. Chinea, J. Marrero, And M. DE Leon, A canonical differential complex for Jacobi manifolds, Michigan J. Math., 45 (1998), pp. 547-579.

[7] C. Di Pietro And A. M. Vinogradov, A spectral sequence associated with a symplectic manifold, Dokl. Akad. Nauk, 413:5 (2007), pp. 591-593.

[8] C. Di Pietro, Sequenza Spettrale assoiata ad una Varietà Simplettica, Ph.D. Thesis, Salerno, 2006.

[9] A. DoLD, Lectures on algebraic topology, Springer-Verlag, 1972.

[10] M. Fernandez, R. Ibanez, And M. De Leon, Poisson cohomology and canonical homology of Poisson manifolds, Archivum Mathematicum, 32 (1996), pp. 29-56.

[11] M. Fernandez, R. Ibanez, And M. De Leon, Coeffective and de Rham cohomologies of symplectic manifolds, J. of Geometry and Phys., 27 (1998), pp. 281-296.

[12] S. I. Gelfand And Iu. I. Manin, Methods of homological algebras, Moscow, Nauka, 1988.

[13] P. Griffith and J. Harris, Principles of algebraic geometry, John Wiley and Sohns, 1994.

[14] F. Guerida And A. Lichnerowicz, Geometrie des algebres de Lie locales de Kirillov, J. Math. Pures et Appl., 63 (1984), pp. 407-484. 
[15] K. Hasegawa and Y. Kamishima, Locally Conformal Kähler Structures on Homogeneous Spaces, arXiv:1101.3693 (To appear in Memorial Volume of Professor Shoshichi Kobayashi, Progress in Mathematics, Springer).

[16] J. L. Koszul, Crochet de Schouten-Nijenhujs et cohomologie, in "Elie Cartan et les Math. d'Aujour d'Hui", Asterique hors-seire, (1985), pp. 251-271.

[17] H. V. Le, P. Somberg, And J. Vanzura, Poisson smooth structures on stratified symplectic spaces, Proceedings in Mathematics \& Statistics, 98 (2015), chapter 7, pp. 181-204, arXiv:1011.0462.

[18] S. Lefschetz, L'Analysis Situs et la Geometrie Algebrique, Gauthier-Villars, Paris, 1924.

[19] Th. N. Lepage, Sur certaines congruences des formes alternaes, Bull. Soc. Roy. Sci., Liege, 15 (1946), pp. 21-31.

[20] A. Lichnerowicz, Les Varietes de Poisson et leurs algebres de Lie associes, J. Diff. Geometry, 12 (1977), pp. 253-300.

[21] V. V. Lychagin, Contact geometry and non linear second-order differential equations, Russian Math. Surveys, 34:1 (1979), pp. 149-180.

[22] C. M. MARLE, On Jacobi manifolds and Jacobi bundles, Symplectic geometry, groupoids, and integrable systems (Berkeley, CA, 1989), pp. 227-246, Math. Sci. Res. Inst. Publ., 20, Springer, New York, 1991.

[23] J. MCCleary, A user's guide to spectral sequence, Cambridge studies in advanced mathematics, v.58, Mathematics Springer, 2001.

[24] D. V. Millionshchikov, Cohomology of solvable Lie algebras and solvmanifolds, Mathematical Notes, 77 (2005), pp. 61-71.

[25] M. Rumin, Formes differentielles sur les varietes de contact, J.D.G., 39 (1994), pp. 281-330.

[26] H. SAWAI, A construction of lattices on certain solvable Lie groups, Topology and its Applications, 154 (2007), pp. 3125-3134.

[27] H. SAWAI, Locally conformal Kähler structures on compact nilmanifolds with left-invariant complex structures, Geom Dedicata, 125 (2007), pp. 93-101.

[28] D. Tischler, On fibering certain foliated manifolds over $S^{1}$, Topology, 9 (1970), pp. $153-154$.

[29] L. S. Tseng And S.-T YAu, Cohomology and Hodge Theory on Symplectic Manifolds, I, II, J. Differential Geom., 91:3 (2012), pp. 383-416 and pp. 417-443, arXiv:0909.5418, arXiv:1011.1250.

[30] I. Vaisman, Locally conformal symplectic manifolds, Internat. J. Math. Math. Sci., 8 (1985), pp. 521-536.

[31] L. Ornea And M. Verbitsky, A report on locally conformal Kähler manifolds, Harmonic maps and differential geometry, pp. 135-149, Contemp. Math., 542, Amer. Math. Soc., Providence, RI, 2011, arXiv:1002.3473.

[32] C. VoIsin, Hodge Theory and Complex Algebraic Geometry, I, Cambridge University Press, 2007.

[33] A. WEIL, Introduction a l'etudé des variétés kähleriennes, Hermann, Paris, 1958.

[34] R. WELLs, Differential analysis on complex manifolds, Springer-Verlag, 2nd edition, 1986.

[35] D. YAN, Hodge structures on symplectic manifolds, Advances in Math., 120 (1996), pp. 143-156. 